\title{
Experimental Investigation of Steady and Unsteady Flow Field Downstream of an Automotive Torque Converter Turbine and Stator
}

\author{
B. V. MARATHE and B. LAKSHMINARAYANA \\ The Pennsylvania State University, Department of Aerospace Engineering, University Park, PA 16802
}

\begin{abstract}
The objective of this investigation is to understand the steady and the unsteady flow field at the exit of an automotive torque converter turbine and stator with a view towards improving it's performance. A high frequency response five-hole probe was designed and built to measure the three-dimensional steady and unsteady flow fields. The measurements were conducted in a stationary frame of reference and the data were processed to derive the flow properties in the relative (turbine) frame of reference. The experimental data were processed in the frequency domain by spectrum analysis and in temporal-spatial domain by ensemble averaging technique. The data show that the flow field is highly unsteady with high unresolved unsteadiness (approx. 17-21\% of mean value) and significant blade-to-blade periodic component ( approx. 6\% of mean value). The unresolved unsteadiness and periodic unsteadiness increase with an increase in the radius from the shell to the core whereas the aperiodic unsteadiness does not show any systematic variation with the radius. The experimental data reveal the presence of a low momentum region near the core due to possible flow separation and reattachment inside the turbine passage. Data also show the presence of strong secondary flow near the core and weak secondary flow near the shell at the exit of the turbine. These secondary flows generate high levels of turbulence. A comparison of the flow properties upstream and downstream of the stator in the stationary frame of reference indicate the presence of high losses near the core due to high turbulence levels and large secondary flows, and high losses near the shell due to possible corner separation near the shell suction surface inside the stator blade passage. The unsteadiness in the flow properties upstream of the stator is high. The rms value of the unsteady total velocity is approx. $20 \%$ of the steady state value. Periodic and aperiodic unsteadiness were also found significant.
\end{abstract}

Key Words: Turbomachinery; Torque converter; Turbine; Unsteady; Five-hole probe

$\mathbf{T}$ The torque converter flow field is very complex. It is three dimensional, viscous and unsteady due to the close coupling between rotor and stator elements. Additional complexities arise due io differential rotor speeds of the pump and the turbine. The unsteadiness is generated due to potential and viscous interactions at the rotor-rotor and the rotor-stator interfaces. The turbine and the pump passages are narrow, with long chord length and are dominated by viscous effects, separation and secondary flow. The nature and magnitude of the unsteadiness varies with several parameters including spacing between two blade rows, number of blades in both the rotor and the stator, thickness of the blade trailing edge, location of flow separation, secondary flows and other geometrical and operating parameters.
Many successful attempts have been made in the past to measure the unsteady flow field inside the turbomachinery elements. However, very few attempts have been made to measure the steady and the unsteady flow field inside the torque converter. Bahr et al. (1990) measured the unsteady flow field in the actual configuration of the torque converter using Laser Doppler Velocimetry. The average intensity in velocity fluctuation at the stator inlet was observed to be 15 to 20 percent of the mean velocity, which is consistent with the present data. Brun et al. (1994) and Gruver et al. (1994) measured the steady and unsteady flow field inside the pump of a torque converter. Their results reveal the presence of strong secondary flows and large velocity fluctuations (approx. 20\%) inside the pump passage. They also found that the 
secondary flow changes direction from the mid-chord to the trailing edge. Similar results were also observed in the computational efforts by By, Kunz and Lakshminarayana (1995). They attributed this phenomenon to the transportation of streamwise vorticity vector in a rotating curved passage. LDV measurements inside the turbine of a torque converter presented by Brun (1993) show a very complex flow field inside the turbine passage. A low velocity zone was observed near the core-suction surface near the leading edge of the turbine blade. The flow reattached near the core-suction surface before the midchord location and the low velocity zone was not observed. However, a low velocity zone was observed near the pressure surface at the mid-chord location indicating a thick boundary layer on the pressure surface at the mid-chord location. At the exit, two regions of low through flow velocity were observed near the core; one near the suction surface and other near the pressure surface. The flow near the shell was nearly uniform from pressure to suction surface. These observations are in agreement with the data presented in this paper. Another attempt, to measure the flow field inside a torque converter, was made by Browarzik and Grahl (1992) using a hot-film probe. The geometrical configuration used was simplified to facilitate the measurements. Also, the operating fluid was water instead of oil. Their frequency spectrum shows very high levels of unsteadiness at different frequencies which is consistent with the data presented in this paper.

The objective of the present investigation is to improve the performance of the torque converters through a detailed insight into the flow phenomenon in each blade row. The thrust of this paper is to understand the steady and the unsteady flow field at the exit of the turbine and the stator at maximum efficiency operating conditions. The steady flow at the exit of the stator is presented by Marathe, Lakshminarayana and Dong (1994).

\section{FACILITY AND INSTRUMENTATION}

The torque converter test facility used in this research program is shown in Fig. 1. Detailed description of the experimental facility and static pressure distribution on the stator blade is given by By and Lakshminarayana (1991, 1995). This facility has five components: driver and absorption dynamometer, test section, oil system and control system. The driver dynamometer is a $30-\mathrm{HP}$ DC motor that delivers the precise amount of torque or rpm specified by the control system and drives the pump. The turbine is connected to the absorption dynamometer, which is controlled to maintain constant torque or rpm.

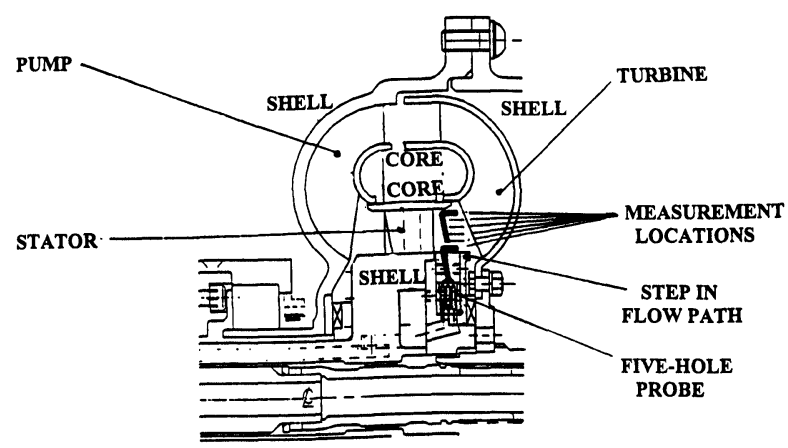

BLADE ANGLES AND VELOCITY TRIANGLES AT SR=0.8

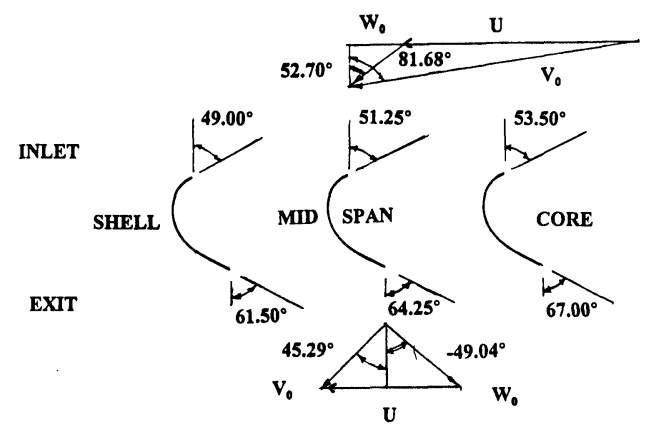

FIGURE 1 Experimental Facility, Measurement Locations and Blade Angles.

The cross section of the torque converter flow path is an elliptical torus formed by pump, turbine and stator blades. The inner boundary of the torus is called the core and the outer boundary is called the shell. It has been observed that high oil pressure prevents the cavitation inside the blade rows, and maintaining elevated temperatures enables stable flow conditions with repeatable performance. Hence, an oil system is used to keep the oil pressure and temperature constant at $14 \mathrm{psi}$ and $60^{\circ} \mathrm{C}$, respectively. The performance of a torque converter is expressed in terms of torque ratio (TR) and speed ratio (SR), defined in the nomenclature.

Tests were conducted at $\mathrm{SR}=0.0,0.2,0.4,0.6$ and 0.8 to simulate all the conditions the torque converter experiences in actual automatic transmission. The design speed ratio of the torque converter is 0.6 . It has a maximum efficiency at $\mathrm{SR}=0.8$ and minimum efficiency at $\mathrm{SR}=0.0$. The data acquired at the exit of the turbine at $\mathrm{SR}=0.8$ is presented and interpreted in this paper. The blade geometry, blade angles and inlet flow angle, (obtained from one-dimensional analysis) at this speed ratio, are shown in Fig. 1. Measurements are carried out at 6 radial positions and 1 tangential position at 0.4276 stator axial chords upstream of the stator, (or 0.068 turbine core section chord downstream of the turbine 
trailing edge and 0.203 turbine shell section chord downstream of the turbine trailing edge) as shown in Fig. 1. The non-dimensional radial position at the turbine shell and core will be designated by $\mathrm{H}=0.0$ and 1.0 respectively.

A miniature fast-response five-hole probe $(1.67 \mathrm{~mm}$ tip diameter) was designed and fabricated to facilitate the measurements of the flow properties upstream and downstream of each of the blade rows. Details of the five-hole probe are shown in Fig. 2. Five miniature pressure transducers were mounted inside the five-hole probe. This allowed extremely small cavity volume between the probe tip and the pressure transducer. The cavity between the probe tip and the pressure transducer was filled with oil to improve the response time of the probe. The tests performed in a hydroacoustic chamber indicate a frequency response of approximately $8 \mathrm{KHz}$. This frequency response was considered sufficient since the maximum blade passing frequency is $585 \mathrm{~Hz}$. The validation of the five-hole probe for measurement of unsteady flow field will be presented in another paper in preparation. A pressure tap was installed on the stator shell surface (hub) for measuring the hub static pressure. This static pressure was used for normalizing the stagnation and static pressures measured by the five-hole probe upstream and downstream of the stator.

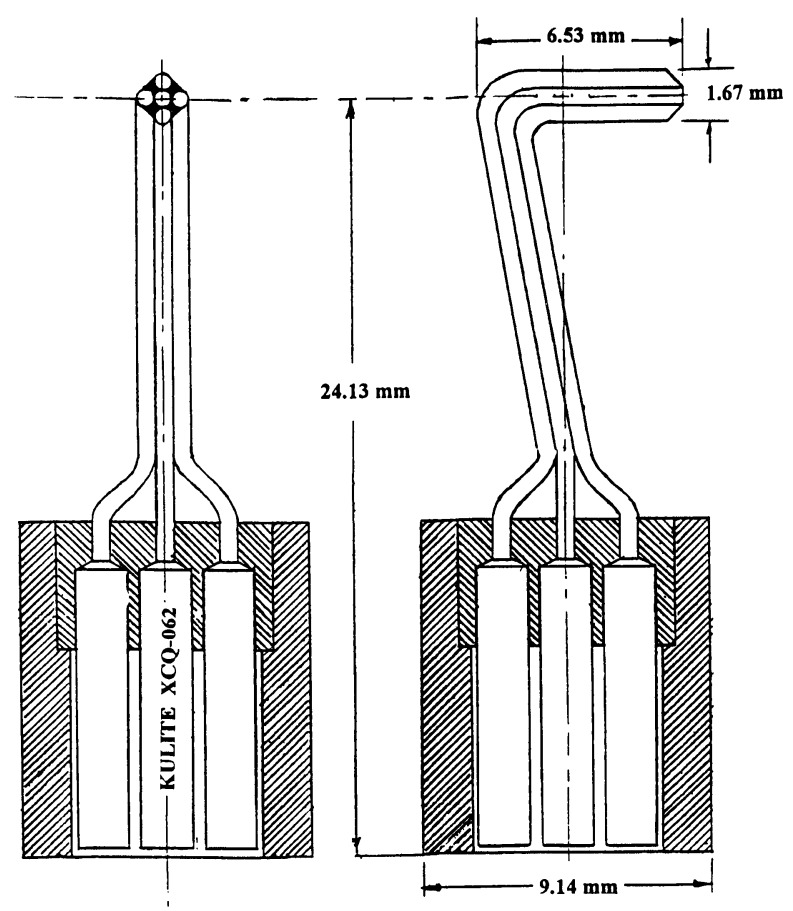

FIGURE 2 Transient Five-Hole Probe.
The signals from the pressure transducers were routed through the recess in the stator shaft to a common-mode signal amplifier outside the test section. The amplified signal was acquired and recorded by the DAS-50 data acquisition system. Maximum data acquisition rate of the DAS-50 system is $1 \mathrm{MHz}$. However the data was acquired at 50 times the blade passing frequency (approx. $25 \mathrm{KHz}$ for SR 0.8) for both the pump and the turbine blades to obtain 50 points in each passing blade passage. A signal from the shaft encoder, mounted on the turbine shaft, was used as clocking frequency to maintain the data acquisition rate precisely at 50 times the blade passing frequency of the turbine. The data acquisition frequency data was downloaded from the DAS-50 memory buffer to a PC-486 where it was processed in both the frequency and the temporal-spatial domain. Two static pressures on the stator were used as reference pressures for normalizing the total and the static pressures obtained from the five-hole probe. A thermocouple was installed on the hub of the stator for accurate reference temperature. The probe calibration has an accuracy of $\pm 1^{\circ}$ in angle measurement. The pressure measurements are obtained to an accuracy of \pm 0.01 psi. The transducer drift introduces a maximum error of $0.5 \%$. This results in a maximum $1-2 \%$ error in the velocities and $0.5 \%$ error in the static and total pressures. Corrections to the probe due to the blockage and the pressure gradient are incorporated into the data processing. The maximum cumulative error in the velocity and pressure measurements is estimated to be $2-3 \%$.

\section{SIGNAL PROCESSING AND AVERAGING TECHNIQUE}

The method of deriving $\mathrm{P}_{0}, \mathrm{P}_{\mathrm{S}}, \alpha, \beta$ from the five-hole probe data is described by Treaster and Yocum (1979). The unsteady pressure data from the five-hole probe is processed to obtain the instantaneous values of $\mathrm{P}_{0}, \mathrm{P}_{\mathrm{S}}, \alpha$, $\beta$. The data was processed using two different methods described later in the paper. The signals from the pressure transducers, connected to the five-hole probe, were processed digitally and also through a spectrum analyzer to determine the dominant frequencies. The dominant frequency was found to be that due to the turbine blade passing frequency. It is beyond the scope of this paper to discuss the details of the spectrum analysis.

The nature of unsteadiness can be classified as deterministic and non-deterministic or unresolved. The deterministic unsteadiness is generated by the rotor-stator or the rotor-rotor interactions and is normally periodic over one revolution/cycle. The deterministic unsteadiness can 
be further divided into two categories. 1) Passage-topassage periodic fluctuations 2) Passage-to-passage aperiodic fluctuations. The passage-to-passage periodic fluctuations are caused by the variation of flow properties from the suction to the pressure surface in the upstream blade passages. The passage-to-passage aperiodic fluctuations are caused by the fluctuations at shaft frequencies and also due to variations in the passage-to passage flow due to 1) manufacturing defects 2) misalignment between the stator and the rotors 3) vibrations due to eccentricity of the shaft and 4) unsteadiness induced by the oil pump. The periodic unsteadiness is caused by viscous and potential interactions. Viscous interactions change the magnitude and direction of absolute flow velocity and hence the incidence angle upstream of the stator blade row. The incidence angle to the stator differs from the design, and it is also unsteady. Hence, the stator operates not only at off design inlet angles but also with considerable unsteady flow. The unresolved or nondeterministic unsteadiness is generated by free-stream turbulence and is normally random in nature. However the unresolved unsteadiness referred to in this paper includes random unsteadiness due to turbulence and other non-deterministic/random unsteadiness generated by sources not periodic over one cycle.

The signal from the pressure transducer was decomposed to deterministic and unresolved components. Since, the deterministic signal is generated by the relative motion between the stator and the rotor blade passages, it repeats over every revolution in conventional turbomachinery. However, for the torque converter the pump and the turbine rotors have different rotational speeds. As a result, flow conditions do not repeat over one revolution of any rotor. Hence, it was necessary to find the period over which the deterministic part of the signal repeats itself. At $\mathrm{SR}=0.8$. the pump and the turbine shaft speeds (revolutions per second) are in 5:4 proportion. Hence, five revolutions of pump and four revolutions of the turbine are completed in identical time period. Since the rotational speeds are maintained with high accuracy (less than $\pm 0.05 \%$ deviation in $\mathrm{rpm}$ ), it can be assumed that the deterministic part of the signal will repeat after 5 revolutions of pump or 4 revolutions of the turbine. It should be noted that for speed ratios 0.6 , $0.4,0.2$ and 0.0 the pump and turbine shaft speeds are in 5:3, 5:2, 5:1 and 5:0 proportions respectively. Therefore, the flow conditions (and relative positions of the blade) would be identical after five revolutions of the pump. Time required to complete five revolutions of the pump will be referred to as "cycle" in this paper. Hence, all deterministic signals are considered repeatable over every cycle. Hence, ensemble averaging technique was employed over one cycle instead of one revolution. The ensemble average technique used by Suder et al. (1987) for LDV measurements inside a compressor was modified to incorporate once per cycle averaging in the torque converter. The technique was also adapted to process the five-hold probe data using continuous averaging technique instead of statistical averaging technique used for LDV data. The modified averaging procedure is as presented below.

The pressure signal from the transducer can be divided into four parts.

$$
P_{I J K}=\bar{P}+\hat{P}_{I}+\tilde{P}_{I J}+P_{I J K}^{\prime}
$$

Where, $P_{I J K}$ is the instantaneous pressure signal measured by the five-hole probe, $\bar{P}$ is the temporal average of the signal, $\hat{P}_{I}$ is the passage-to-passage periodic component, $\tilde{P}_{I J}$ is the passage-to-passage aperiodic component and $P_{\mathrm{IJK}}^{\prime}$ is the unresolved component. It should be noted that the subscript ' $\mathrm{I}$ ' is used for all the points in one blade passage, the ' $\mathrm{J}$ ' subscript is used for all the blade passages in one cycle and ' $\mathrm{K}$ ' subscript is used for all the cycles in one data set. Passage-to-passage periodic component $\hat{P}_{\mathrm{I}}$ is generated by the blade passing frequency under consideration. Passage-to-passage aperiodic component, $\tilde{P}_{I J}$ is due to the deterministic signal at frequencies other than blade passing frequency, and unresolved component $P_{I J K}^{\prime}$ includes random fluctuations and other fluctuations related to noise and vibration.

The temporal averaging is performed by taking the average of all the data points. That is,

$$
\bar{P}=\frac{1}{N_{c y c}} \frac{1}{N_{b p c}} \frac{1}{N_{p p b}} \sum_{K=1}^{N_{c y c}} \sum_{J=1}^{N_{b p c}} \sum_{I=1}^{N_{p p b}} P_{I J K}
$$

Where, $\mathrm{N}_{\text {cyc }}$ is the number of cycles per data set (1 cycle $=5$ revolutions of the pump), $\mathrm{N}_{\mathrm{bpc}}$ is the number of blade passages per cycle and $\mathrm{N}_{\mathrm{ppb}}$ is the number of points per blade passage.

The averaging was performed by calculating passageto-passage periodic component by averaging pressure signal over number of blade passages. The equation used is given by.

$$
\hat{P}_{I}=\frac{1}{N_{c y c}} \frac{1}{N_{b p c}} \sum_{K=1}^{N_{c y c}} \sum_{J=1}^{N_{b p c}}\left(P_{I J K}-\bar{P}\right)
$$

The passage-to-passage aperiodic fluctuating component is then calculated by subtracting the respective periodic component from the instantaneous pressure 
signal $\mathrm{P}_{\mathrm{IJK}}$ and then averaging the remaining part over number of cycles. The equation is given by.

$$
\tilde{P}_{I J}=\frac{1}{N_{c y c}} \sum_{K=1}^{N_{c c c}}\left(P_{I J K}-\bar{P}-\hat{P}_{I}\right)
$$

The unresolved pressure component is then calculated by subtracting both the passage-to-passage aperiodic and the passage-to-passage periodic component from the instantaneous pressure. The equations used.are:

$$
\begin{gathered}
P^{\prime}{ }_{I J K}=P_{I J K}-\bar{P}-\hat{P}_{I}-\tilde{P}_{I J} \\
\left(P^{\prime}{ }_{I J K}\right)_{R M S}=\sqrt{\frac{1}{N_{c y c}} \frac{1}{N_{b p c}} \frac{1}{N_{p p b}} \sum_{K=1}^{N_{c c c}} \sum_{J=1}^{N_{b p c}} \sum_{I=1}^{N_{p p b}}\left(P_{I J K}^{\prime}\right)^{2}}
\end{gathered}
$$

Two different approaches were used for processing the data to derive the mean, steady (in the relative frame) and fluctuating velocities. In the first method, the instantaneous pressure signals are processed to obtain instantaneous flow parameters $\left(\mathrm{P}_{0}, \mathrm{P}_{\mathrm{s}}, \alpha, \beta, \mathrm{V}\right.$ etc. $)$. The instantaneous flow parameters are then processed to obtain the mean, periodic, aperiodic and unresolved components of each flow parameter. However this data processing method cannot incorporate spatial error (finite dimensions of the probe) correction. As a result, the quantities obtained by this method could have a large spatial error in the highly unsteady flow field. This method is probably accurate for deriving unresolved unsteadiness. It should be noted that, due to high levels of unresolved unsteadiness, approximately $10 \%$ points were out of the probe calibration range and were eliminated during the averaging process. As a result, the number of averages at each relative measurement location was different. This also introduced error in the ensemble averaged values as the ensemble averaged values near the calibration limits were eliminated due to the unresolved component.

In the second method, the instantaneous pressure signals were processed to obtain ensemble (phase lock) averaged values of the pressure, removing the unresolved component from the pressure signal. The ensemble averaged values were processed with a correction for spatial error to obtain the ensemble averaged values of each flow parameter $\left(\mathrm{P}_{0}, \mathrm{P}_{\mathrm{S}}, \alpha, \beta, \mathrm{V}\right.$ etc. $)$. These values are then processed to obtain mean, periodic and aperiodic components of each flow parameter. Since the unresolved pressure component is removed from the instantaneous pressure signal, the values remain in the calibration range. Hence, less than $0.1 \%$ points were eliminated in the averaging procedure. Thus, the number of averages for each relative measurement position were constant. Also, the spacial error could be corrected in this method. Hence, this method was thought to be more accurate for obtaining periodic and aperiodic components. The data presented in this paper has been processed by using the second method.

The passage-to-passage distribution of the important flow parameters, as well as the radial distribution of the steady and unsteady flow properties at the exit of the entire turbine passage are discussed in the sections that follow. Since the data obtained from the five-hole probe was in the absolute (stator, stationary) reference frame, it was processed to obtain distribution of the flow properties in the relative (turbine, rotational) reference frame. The tangential velocity at each radial location, in the rotating frame of reference, was derived using the equation $W_{\theta}=U-V_{\theta}$ where, $U$ is the blade speed at the measurement radius and $V_{\theta}$ is the absolute tangential velocity component measured by the five-hole probe. Using values of $W_{\theta}, W_{X}$ and $W_{R}$, the value of total relative velocity, relative stagnation pressure and relative yaw angle were obtained. All the velocities, in both the relative and the absolute frames of reference, were normalized by $\mathrm{V}_{\mathrm{REF}}$. The pressures were normalized by reference dynamic head $\mathrm{Q}_{\mathrm{REF}}$.

\section{TURBINE EXIT FLOW IN RELATIVE REFERENCE FRAME}

A comparison of the distribution of the ensemble averaged relative total velocity in approximately three consecutive blade passages is shown in Fig. 3. The flow is nearly periodic with aperiodicity in certain regions. The relative positions of low and high momentum regions are periodic. However, the shape and size of these regions change from passage-to passage. It should be noted that the relative position of the pump blade passage is different by 0.141 passage widths for each consecutive turbine passage. As a result, the inlet flow in each blade passage is significantly different for each passage. This induces aperiodicity in the turbine exit flow field. The magnitude of the aperiodic component is comparable to the magnitude of the periodic component. However, the study of aperiodic flow properties is beyond the scope of this paper. Hence, passage-to-passage distribution of only periodic values is presented in the paper. However, the radial distribution of rms of aperiodic values is presented for comparison with the rms of periodic and unresolved components. Since the flow field under consideration is the turbine exit flow field, the properties are 

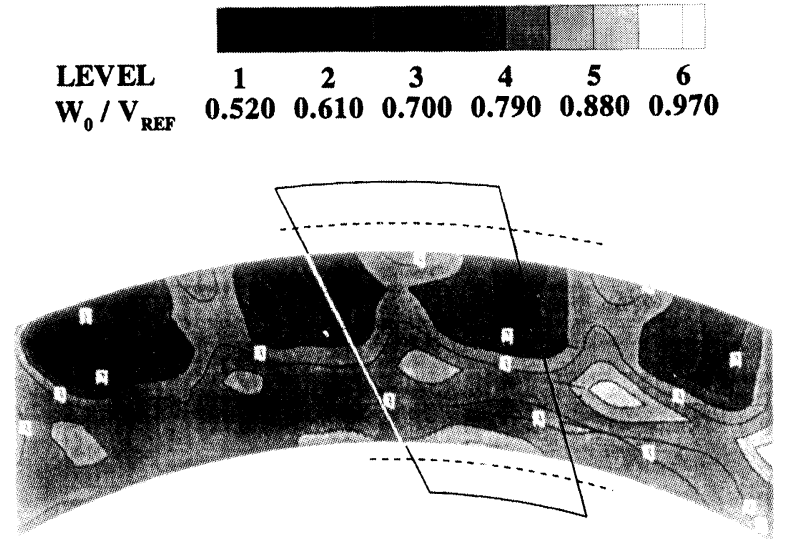

FIGURE 3 Contours of Ensemble Averaged Relative Normalized Total Velocity at Turbine Exit (in Approximately Three Blade Passages).

presented only in the relative (turbine) frame of reference. However, the radial distributions of the mass averaged quantities are presented in both the relative and the absolute frame of reference to show the radial variation of the flow properties as observed by the turbine and by the stator.

\section{Stagnation Pressure Coefficient $\left(\hat{C}_{P O}\right)$ at Turbine Exit}

The distribution of relative stagnation pressure coefficient $\left(\hat{C}_{P O}\right)$ is shown in Fig. 4. The tangential distribution of relative stagnation pressure near the shell $(\mathrm{H}=0.149$ to 0.396 ) shows that tangential variation in the stagnation

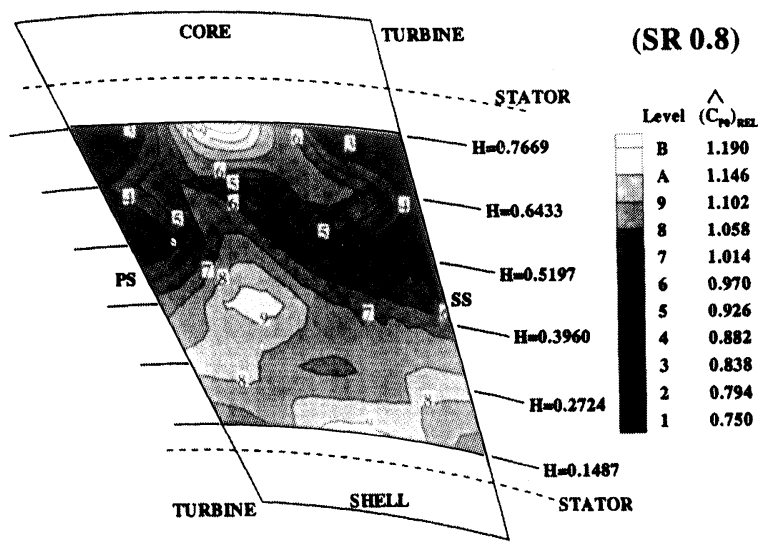

FIGURE 4 Contours of Relative Total Pressure Coefficient $\left(\hat{C}_{P O}\right)_{R E L}$ at the Turbine Exit. pressure coefficient is small. It should be noted that the distance between the probe and trailing edge of the turbine decreases with an increase in the radius (see Fig. 1). Hence, at lower radii (near shell) larger downstream distances between the probe and the turbine blade trailing edge cause larger wake decay and this results in smaller variations in the flow properties. Also, the flow field geometry near the turbine shell has a step in the flow path as shown in Fig. 1. This step causes rapid flow mixing and diffuses the endwall boundary layers near the shell. As a result, the flow near the shell is nearly uniform. The variation of relative stagnation pressure in tangential direction is high near the core region, where a region of low stagnation pressure is observed close to the wakes. This region extends from $\mathrm{H}=0.520$ to 0.767 . This low stagnation pressure region corresponds to the region of low relative velocities discussed later in the paper. The low velocity zone could be attributed to the separation and reattachment of the flow inside the turbine blade passage near the core-suction surface corner. Also, the passage vortex near the core generates a low momentum region in the center of the vortex. This low momentum region can be observed in the mid-passage region.

In addition, a region of high relative stagnation pressure from $\mathrm{H}=0.643$ to 0.767 can also be observed near the mid-passage in Fig. 4. This may have been caused by acceleration of the flow due to the blockage generated by the low momentum region at $\mathrm{H}=0.520$ to 0.643 . The axial velocities in this region are found higher than those near the wake, as explained later.

The low relative stagnation pressure region near the core coincides with the low absolute stagnation pressure region (not shown). These low absolute stagnation pressures are attributed to the lower values of the static pressure and the axial velocity in that region as discussed later in the paper. A region of low absolute stagnation pressure is also observed at $\mathrm{H}=0.396$ which coincides with the region of high relative stagnation pressure. This indicates that the high velocity jet in the relative frame is perceived as a low momentum region in the absolute frame of reference due to high values of axial and relative tangential velocities in that region. This also explains the lower values of $\left(\hat{C}_{P O}\right)_{A B S}$ near the shell and higher values near the core.

The radial distribution of the mass averaged relative stagnation pressure coefficient $\left(\overline{C_{P O}}\right)_{R E L}$ shows rather smooth distribution from shell to $\mathrm{H}=0.396$ (Fig. 5), with a sudden decrease from $\mathrm{H}=0.396$ to 0.520 and a gradual increase towards the core from $\mathrm{H}=0.520$. The low $\left(\overline{C_{P O}}\right)_{R E L}$ region corresponds to the low pressure core region shown in Fig. 4 . The values of $\left(\overline{C_{P O}}\right)_{R E L}$ are generally lower from the mid-span to the core. 


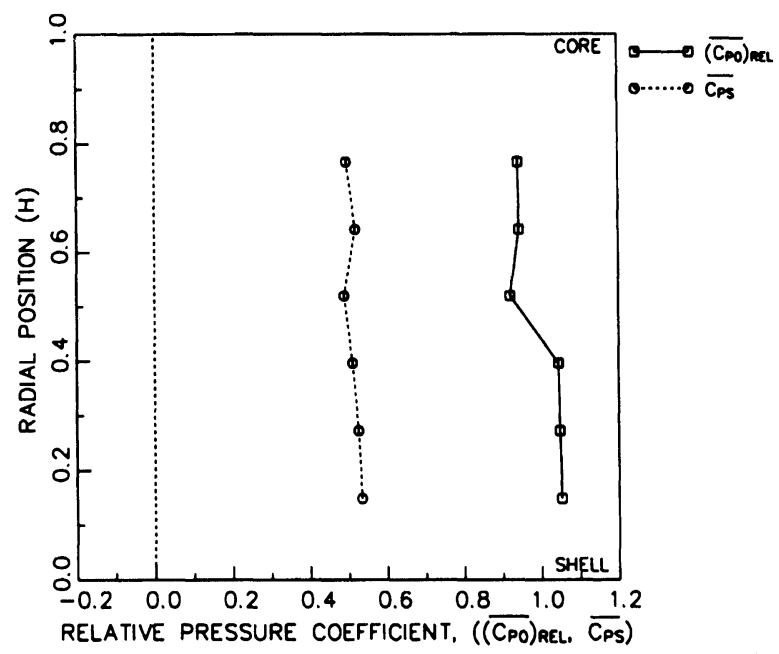

FIGURE 5 Radial Distribution of Mass Averaged Pressure Coefficients $\left({\overline{\left(C_{P O}\right)_{R E L}}}_{1}, \bar{C}_{P S}\right)$ at the Turbine Exit.

\section{Static Pressure Coefficient $\left(\hat{C}_{P S}\right)$ at Turbine Exit}

The distribution of static pressure coefficient (Fig. 6) at the exit of turbine passage shows nearly uniform distribution of $\left(\hat{C}_{P S}\right)$ near the shell. Lower static pressures are observed at mid-passage and higher inside the blade wakes at both the shell and the mid-span region. This is consistent with the static pressure distribution in the typical turbine blade rows where higher static pressures are observed inside the blade wake. However, near the core region the static pressure is maximum at midpassage and minimum near the wake regions. One possible cause for this type of distribution is that the flow field at the exit of the turbine in the core region is fully

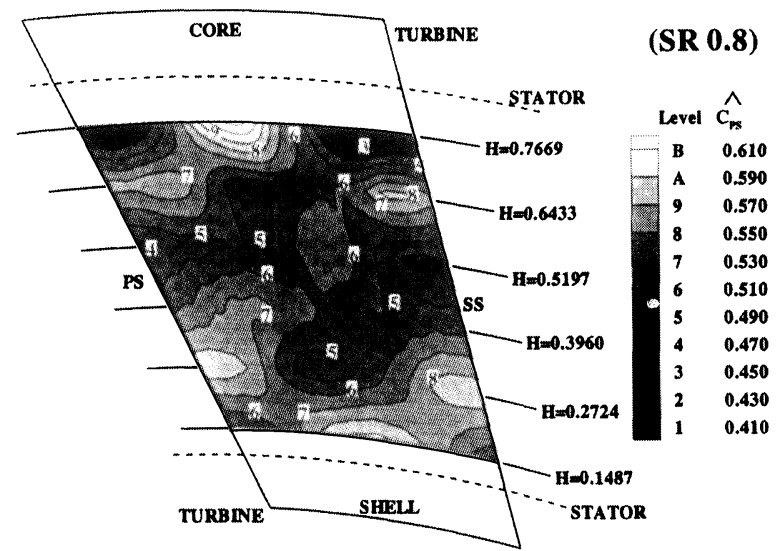

FIGURE 6 Contours of Static Pressure Coefficient $\left(\hat{C}_{P S}\right)$ at the Turbine Exit.

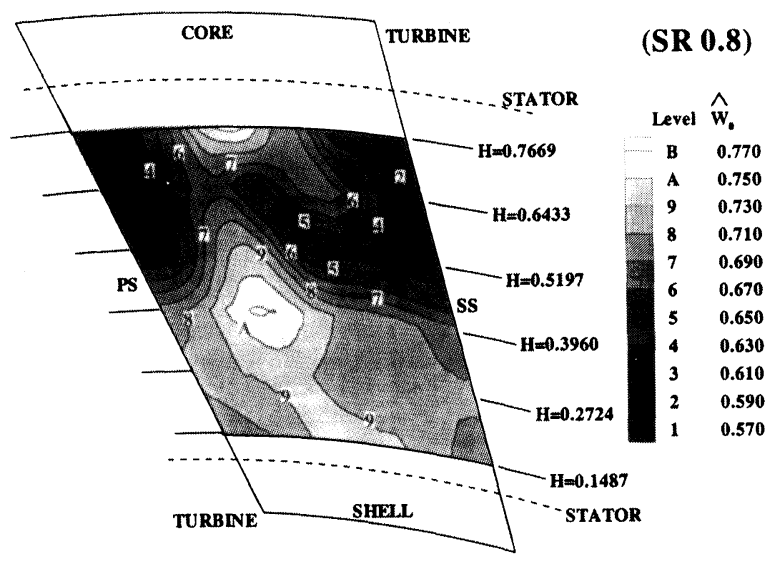

FIGURE 7 Contours of Relative Normalized Total Velocity $\left(\hat{W}_{o}\right)$ $\left.V_{R E F}\right)$ at the Turbine Exit.

developed and hence the static pressure distribution inside the turbine blades is different from the conventional turbomachinery blade rows.

The distributions of $\left(\hat{C}_{P O}\right)_{R E L}$ and $\left(\hat{C}_{P S}\right)$ near the core are very similar. The flow at the mid-passage near the core is dominated by blockage effects caused by low momentum region near the wakes. The acceleration of the flow causes higher relative velocity as seen in Fig. 7 and lower absolute velocity as seen in Fig. 8. This results in the lower static pressure drop (or higher static pressure in this region). On the other hand, the region dominated by the separation (inside the turbine blade) has lower relative velocity and higher static pressure drop (or lower static pressure). The region dominated by the secondary vortex also shows lower $\left(\hat{C}_{P S}\right)$ values at the center of the vortex. This is consistent with the observations made by Zaccaria and Lakshminarayana (1995).

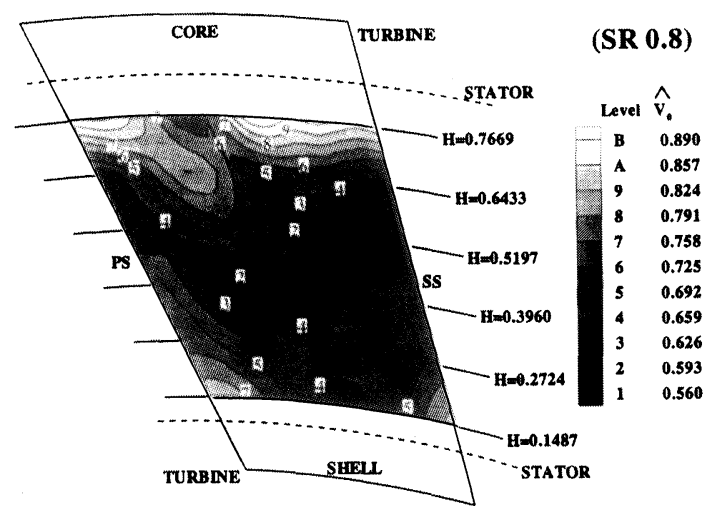

FIGURE 8 Contours of Absolute Normalized Total Velocity $\left(\hat{V}_{0} /\right.$ $\left.V_{R E F}\right)$ at the Turbine Exit. 
Radial distribution of mass averaged static pressure coefficient $\left(\overline{C_{P S}}\right)$ (Fig. 5) shows a smooth variation of static pressure. The static pressure decreases with an increase in the radius. This could be attributed to the curvature of the streamlines in meridional as well as in azimuthal directions as explained by Marathe, Lakshminarayana and Dong (1994).

\section{Velocity Profiles at the Turbine Exit}

The distribution of non-dimensionalized relative total velocity behind the turbine $\left(\hat{W}_{01}\right)$ is shown in Fig. 7. The velocity distribution is nearly uniform near the shell with only $7 \%$ variation across the blade passage. The wakes are not well defined. The turbine wakes are thin due to small blade thickness and the flow mixes rapidly downstream of the turbine blade. Also the extended stator hub generates a step in the flow path near the shell (see Fig. 1). This step enhances the flow mixing and influences the streamline structure near the shell. As a result, the wake decays rapidly and the velocity distribution is uniform near the shell. A region of low total velocity is observed near the core which extends from the pressure to the suction surface and from $\mathrm{H}=0.55$ to $\mathrm{H}=0.88$. The low velocity region near the suction and the pressure side of the wake can be attributed to the flow separation and reattachment of the flow inside the turbine blade and also to the larger boundary layer growth on the pressure surface as observed by Brun (1993) at mid-chord of the turbine blade. The measurements by Brun (1993) confirm the presence of a low momentum region near the core-suction surface due to possible corner separation near the leading edge of the turbine rotor, and reattached flow with the low velocity region near the core-suction surface at the trailing edge of the turbine. Another possible cause of the low velocity zone is the presence of the passage vortex near the core discussed later in the paper. As mentioned earlier, the higher velocity near the mid-passage of the core region and also near the midpassage near $\mathrm{H}=0.396$ is caused by flow acceleration due to the blockage caused by the low momentum regions near $\mathrm{H}=0.520$ to 0.646 .

The distribution of the absolute total velocity is shown in Fig. 8. The contours show a low velocity region near $\mathrm{H}=0.520$ near the suction surface. This is consistent with the low absolute stagnation pressure coefficient in this region (not shown). This low velocity region could be attributed to the low axial velocities in this region. A low velocity region is also observed near $\mathrm{H}=0.396$ near the pressure surface. This can be attributed to the smaller values of $\left(\hat{V}_{\theta}\right)$ caused by high values of $\left(\hat{\mathrm{W}}_{\theta}\right)$ and lower values of $U$ at that radius. This also explains the presence of higher values of velocities near the core and lower values of velocities near the shell and mid-span.

Radial distribution of the mass averaged non-dimensional relative total velocity $\left(\overline{W_{01}}\right)$ (Fig. 9) shows uniform distribution near shell with a small drop near mid-span region. This drop in the relative total velocity can be attributed to an approximately 20\% drop in the axial velocity and approximately $10 \%$ drop in the relative tangential velocity at this location. The drop in the axial relative tangetial velocity can be atributed to the low relative velocity zone discussed earlier in the paper. The drop in the relative tangetial velocity results in an increase in the absolute tangential velocity. As a result, the radial distribution of mass averaged abolute total velocity is smooth. It should be noted that although measurements cover only $60 \%$ of the turbine passage span, they cover approximately $82 \%$ of the stator passage span. Since the endwall boundary layers from the turbine blade are convected to the stator blade passage, the effects of the endwall boundary layers should be noticeable at the measurement locations. However, the effects of either the shell or the core endwall boundary layer were not observed in either the relative or the absolute total velocity distribution at the stator inlet.

The absolute total velocities, shown in Fig. 8, are lower near the mid-span $(H=0.520)$ and higher near the shell and the core. This could be attributed to two possible explanations: 1) The boundary layers on the endwalls of the turbine are thin due to the accelerating flow inside the blade passage. The flow separation does

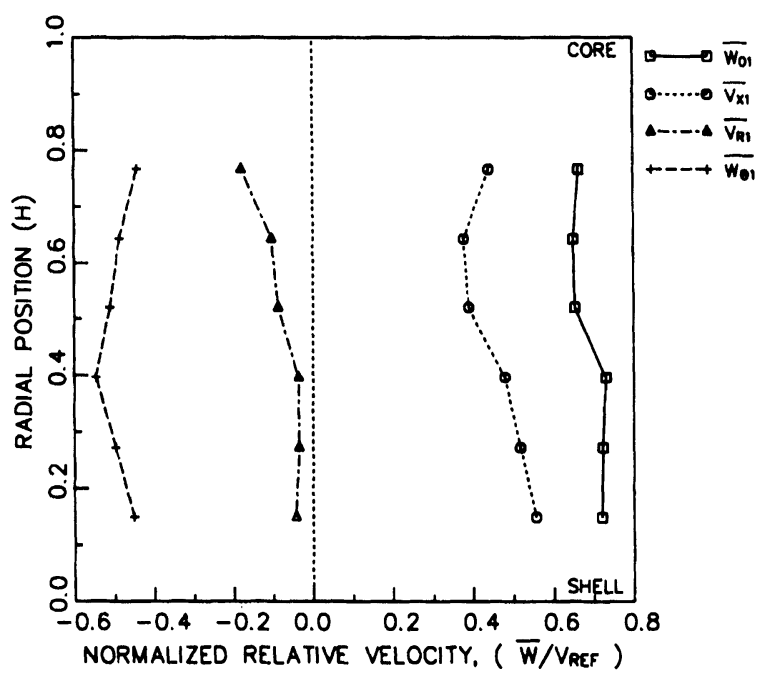

FIGURE 9 Radial Distribution of Mass Averaged Normalized Velocity Components $\left(\bar{W} / V_{R E F}\right)$ at the Turbine Exit. 
not occur near the shell endwall. A small corner separation occurs near the core endwall inside the turbine blade passage. However, the flow reattaches and the low momentum region generated by the flow separation is transported towards the mid-span region due to strong secondary flows. 2) The shell boundary layer is mixed due to a step in the flow path geometry before it reaches the five-hole probe location and the core boundary layer is partially mixed with the secondary flows and partially bled into the core region through the gap in turbine and stator cover. This also explains the small amount of radial outward flow near the core observed in the radial distribution of the mass averaged radial velocity shown in Fig. 9, and discussed later in the paper.

Blade-to-blade distribution of axial velocity (Fig. 10) clearly shows a low velocity zone near the core suction surface, where axial velocities are 50\% lower than those near the shell. The higher axial velocities near $\mathrm{H}=0.767$ can be attributed to the presence of secondary flows in the core region and the blockage effect caused by the low momentum fluid region near $\mathrm{H}=0.520$. The axial velocity distribution is nearly uniform from the pressure side to the suction side near the shell. This could be attributed to the high flow mixing near the shell. It should be noted here that the flow near the shell remains attached to the blade surface inside the turbine as observed by Brun (1993). It should also be noted that although the secondary flow is strong near the core the axial velocities do not show low velocities at the center of the vortex.

The passage-mass averaged axial velocity at the inlet of the stator was found to be $0.07 \%$ smaller than that measured by Marathe, Lakshminarayana and Dong (1994) at the exit of the stator. The radial distribution of

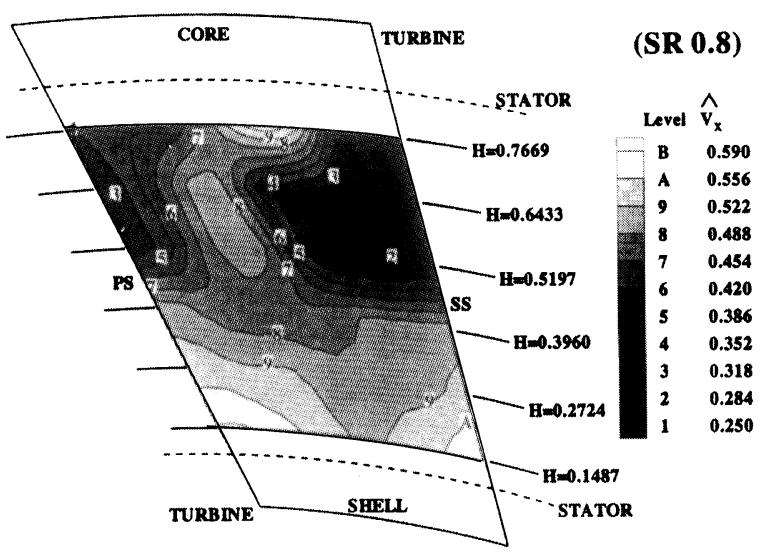

FIGURE 10 Contours of Normalized Axial Velocity $\left(\hat{V}_{x} / V_{R E F}\right)$ at the Turbine Exit. the mass averaged axial velocity (Fig. 9) shows an almost linear variation in the axial velocity from $\mathrm{H}=$ 0.06 to 0.39 . The gradient in the axial velocity in this region is identical to the gradient in the axial velocity in the corresponding region downstream of stator reported by Marathe, Lakshminarayana and Dong (1994). This indicates that redistribution of mass flow inside the stator near the shell is small. Low axial velocities from $\mathrm{H}=$ 0.520 to $\mathrm{H}=0.643$ are caused by the low momentum core flow region discussed earlier. The secondary flows and blockage due to the low momentum fluid region redistribute the mass flow near the core increasing the mass averaged axial velocity at $\mathrm{H}=0.767$. This observation is consistent with the data acquired by Brun (1993).

\section{Yaw Angle $(\hat{\beta})$ Profiles at Turbine Exit}

The contours of the yaw angle (measured from the axial direction) are shown in Fig. 11. As indicated in Fig. 1, the blade outlet angle is -61.5 degrees at the shell and -67 degrees at the core. The distribution of yaw angles is nearly uniform near the shell except near the wakes. The flow is substantially underturned in this region, with higher underturning occurring near the wakes. This is consistent with the higher axial velocities observed in this region. A high flow turning region is observed near the core which corresponds to the region of low axial velocity discussed earlier in the paper. The high flow turning region is attributed to the presence of strong secondary flow in the core region. A small region of flow overturning is observed from $\mathrm{H}=0.520$ and 0.643 close to the wake regions. The flow is substantially under

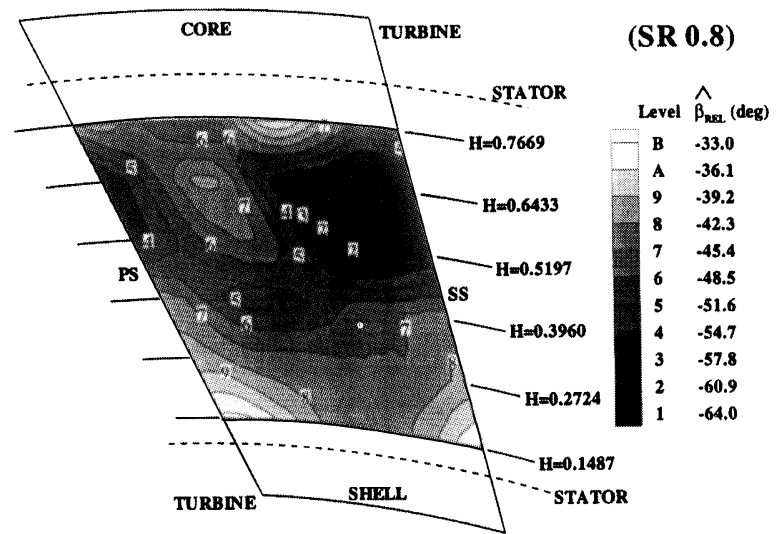

FIGURE 11 Contours of Relative Yaw Angle $\left(\hat{\beta}_{R E L}\right)$, (in degrees) at the Turbine Exit. 
turned in all other regions. The deviation angles calculated from the passage averaged velocities are very high (approx. 20-25 ) near the shell, low near mid-span (approx. 10-13 ${ }^{\circ}$ ) and high (approx. 15-20 $)$ near the core.

Axial flow turbomachinery turbine blades do not show large deviation angles except in the regions of secondary flow as reported by Zaccaria and Lakshminarayana (1995). In the axial flow turbine rotors the flow remains attached to the blade surface and the effect of secondary flow is restricted to the $20 \%$ blade span near hub and tip endwalls. It should be noted that although the five-hole probe measurements were taken at $40 \%$ axial chord upstream of the stator, the thick leading edge of the stator blade generates significant blockage effect at the measurement location (which is approximately 2 leading edge diameters upstream of the stator leading edge). As a result, the flow deviates before it reaches the five-hole probe and the yaw angle measured is higher than the actual yaw angle at the exit of the turbine. The change in the yaw angle due to the deviation of flow caused by the stator leading edge is estimated to be as high as 10 degrees in the relative frame of reference. Hence, the flow deviation angle after the correction is approximately $5-15^{\circ}$ near the shell. It should be noted that the flow turns by approximately $180^{\circ}$ in $\mathrm{x}-\mathrm{r}$ plane and approximately $130^{\circ}$ in $r-\theta$ plane in a turbine passage. Hence, the flow deviation angle is small compared to the overall turning of the flow. The distribution of the yaw angle shows higher yaw angles in the low momentum core flow region. This can be explained as follows: The reattachment of the separated flow on the suction surface near the mid-span deviates the streamlines towards the suction surface causing overturning of the flow in the region of low momentum fluid. Another possibility is that the low momentum fluid region is generated due to the passage vortex and the overturning in the region of low momentum fluid is due to the overturning arm of the passage vortex.

The radial distribution of passage (mass) averaged relative yaw angles (i.e., yaw angles calculated from the mass averaged axial and tangential velocities) is shown in Fig. 12. The flow is underturned at every location, with largest values occurring near the core, and the smallest values near the mid-span region. These values are attributed to the secondary flows and core flow region as explained earlier.

\section{Pitch Angle $(\hat{\alpha})$ Profiles at Turbine Exit}

The contours of the pitch angle $\hat{\alpha}$ are shown in Fig. 13. The distribution of pitch angle shows very large varia-

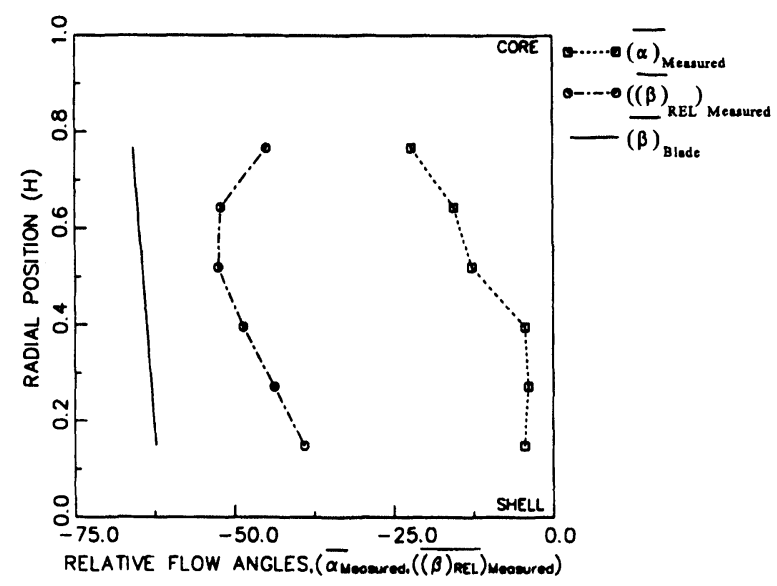

FiIGURE 12 Radial distribution of Mass Averaged Flow Angles $\left(\bar{\alpha}_{\text {Measured }},\left(\overline{(\beta)}_{R E L}\right)_{\text {Measured }}\right)$ at the Turbine Exit.

tions in $\hat{\alpha}$ from the pressure to the suction side and from the core to the shell. The largest variation in the pitch angle is near $\mathrm{H}=0.643$ where pitch angles are as high as 13 degrees near the pressure surface and as high as -49 degrees near the suction surface. The variation is less severe near the shell region. However, a variation from 7 degrees near the blade surfaces to -12 degrees near the mid-passage is observed near $\mathrm{H}=0.273$. These large variations are attributed to strong secondary flows present at the turbine exit. The pitch angle variation is smaller near the shell and the mid-span and larger near the core. This indicates the presence of strong secondary flows near the core and weak secondary flows near the shell. This observation is consistent with the observation by Brun (1993).

The radial distribution of the passage-mass averaged radial velocity (Fig. 9) and the pitch angle (Fig. 12)

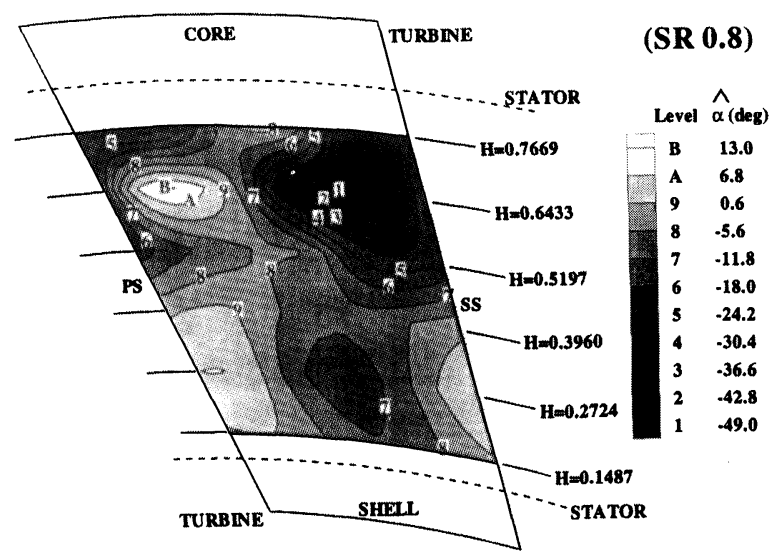

FIGURE 13 Contours of Pitch Angles ( $\hat{\alpha}$ ) (in degrees) at The Turbine Exit. 
shows nearly uniform distribution from $\mathrm{H}=0.149$ to $\mathrm{H}=0.396$ and a smooth increase in the radial velocity from $\mathrm{H}=0.396$ to $\mathrm{H}=0.767$. The radial velocities are very small and negative (inward) near the shell but significant near the core. High magnitudes of radial negative velocities near the core are caused by radially converging flow path from the turbine exit to the stator inlet and by the strong secondary flow near the core. As a result, the streamline angles change from high negative $\left(-25^{\circ}\right)$ near the core to low negative $\left(-5^{\circ}\right)$ near the shell. The uniform distribution of pitch angle near the shell (from $\mathrm{H}=0.149$ to $\mathrm{H}=0.396$ ) is the result of enhanced flow mixing due to the step in the flow path as described earlier in the paper. This observation is consistent with the data by Brun (1993).

\section{Secondary Flow Vectors at Turbine Exit}

The secondary flow vectors (in the relative frame of reference) at the exit of the turbine blade passage are shown in Fig. 14. The secondary flow pattern in the turbine passage is complex due to the presence of meridional, and longitudinal curvature as well as rotation as analyzed by Lakshminarayana and Horlock (1973). The presence of streamwise vorticity at the inlet to the turbine (from the pump) cause the secondary flow to deviate considerably from those observed in isolated rotors or cascades. The secondary flow velocity $\mathrm{W}_{\mathrm{SEC}}$ is defined as

$$
\vec{W}_{S E C}=\vec{i}_{\theta}\left[\left(W_{\theta}\right)_{\text {MEASURED }}-\left(W_{\theta}\right)_{\text {IDEAL }}\right]+\vec{i}_{R} W_{R}
$$

CORE

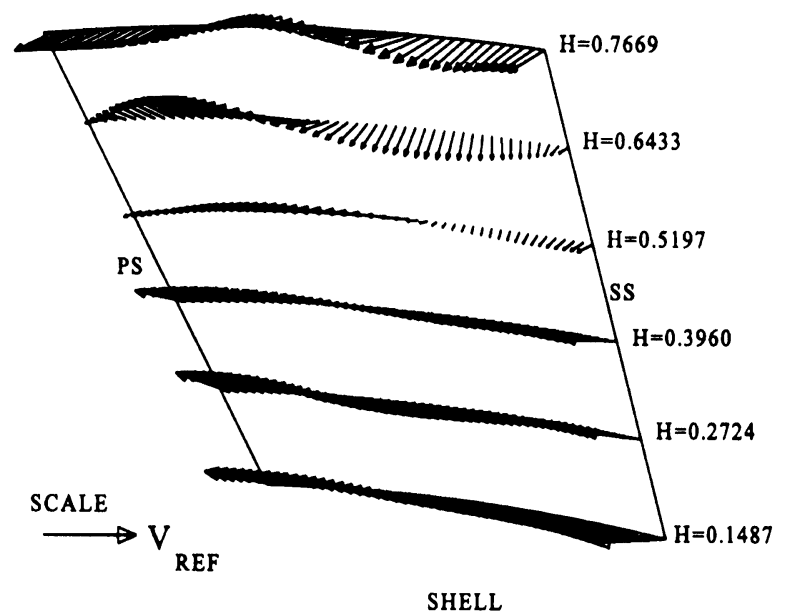

FIGURE 14 Secondary Flow Vectors at the Turbine Exit $\left(\overrightarrow{\mathrm{W}}_{S E C}\right)$.
Where ideal tangential velocity $\left(W_{\theta}\right)_{\text {IDEAL }}$ is calculated by using blade outlet angles as ideal flow outlet angles. In radial and mixed flow turbomachinery it is necessary to account for radial flow direction and calculate the radial component of secondary flow, $\vec{i}_{\mathrm{R}}\left[\left(W_{\mathrm{R}}\right)_{\text {measured }}-\right.$ $\left.\left(W_{\mathrm{R}}\right)_{\text {ideal }}\right]$. However, in the present studies the exact value of the radial component could not be determined. Hence, $\left(W_{\mathrm{R}}\right)_{\text {ideal }}$ was assumed to be zero.

The secondary flow shows presence of a vortex encompassing the entire passage. However, influence of this vortex is weak near the shell and strong near the core. The long flow path and the presence of the step in the flow path at the turbine exit probably decays and modifies the secondary flow considerably near the shell. The core region shows strong secondary vortex which has a clockwise (pressure to suction in the outer part of the vortex) rotation. This is consistent to the passage vortex observed in isolated blade rows and is also consistent with the observations by Brun (1993).

\section{RADIAL DISTRIBUTION OF THE MASS AND TIME AVERAGED FLOW PROPERTIES UPSTREAM AND DOWNSTREAM OF THE STATOR}

The mass averaged flow properties in the absolute frame of reference, at both the inlet and the exit of the stator, are presented in this section. Radial distribution of the mass averaged stagnation $\left(\mathrm{C}_{\mathrm{PO}}\right)_{\mathrm{ABS}}$ and static pressure coefficients $\left(\mathrm{C}_{\mathrm{PS}}\right)_{\mathrm{ABS}}$ upstream and downstream of the stator are shown in Fig. 15. The radial distribution of the absolute stagnation pressure coefficient at the inlet to the stator is smooth with lowest stagnation pressure occurring near $\mathrm{H}=0.520$ and higher stagnation pressures near the core and the shell. The radial distribution of stagnation pressure downstream of stator shows higher stagnation pressures near the mid-span and lower stagnation pressures near the core and the shell. Thus the stagnation pressure loss in the stator blade row is low near mid-span and high (approximately 20-30\%) near the shell and the core. The stagnation pressure rise at mid-span across the stator blade is attributed to the redistribution of the stagnation pressure inside the stator passage. The higher losses near the core and the shell are attributed to the secondary flows, wall boundary layers and possible flow separation in these regions.

The radial distribution of passage-mass averaged static pressure indicates that the static pressure drop through the stator is approximately $0.7 Q_{\mathrm{REF}}$. This pressure drop is much smaller (45\%) than the pressure drop calculated from one dimensional analysis. The discrepancy in the 


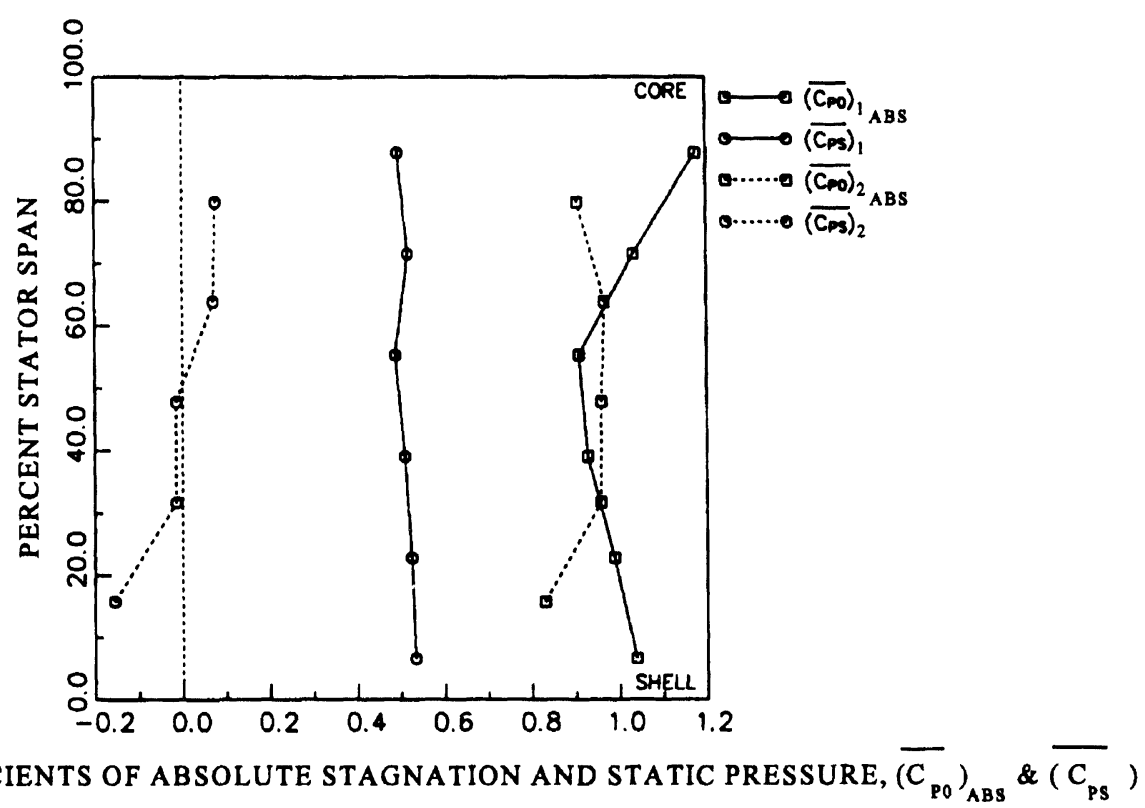

FIGURE 15 Radial Distribution of Mass Averaged Absolute Pressure Coefficients $\left(\overline{C_{P O}}\right)_{A B S},\left(\overline{C_{P S}}\right)$, at the Stator Inlet and Exit

pressure drop arises due to the fact that the stator flow field is highly three-dimensional, turbulent and viscous and also that the one-dimensional analysis cannot predict the flow angles accurately. The static pressure drop is higher near shell than near the core. This is attributed to the differential flow turning in the stator blade. The flow turning is maximum (approx. $17 \mathrm{deg}$.) near the shell and minimum (approx. 8 deg.) near the core. Also, the flow turns in azimuthal direction due to torus flow path geometry. As a result the radial gradient of static pressure is determined by streamline curvature equation. Hence, relative values of $\overline{V_{x}}$ and $\overline{V_{\theta}}$ influence the static pressure distribution.

Radial distributions of the mass averaged absolute velocities are shown in Fig. 16. It should be noted that although the radial distribution of the total velocity distribution in the turbine reference frame is non-uniform, the velocity distribution in the stator reference frame is smooth. Comparison of the total velocities upstream and downstream of the stator show very small acceleration of the flow near the core and high acceleration near the shell. This is consistent with the flow turning angles (Fig. 17) which show higher flow turning near the shell and lower flow turning near the core. The mass averaged axial velocities (Fig. 16) upstream and downstream of the stator show identical distribution from shell to mid-span. However, the axial velocities upstream are lower than the axial velocities downstream from $55 \%$ to $71 \%$ of the blade span. This can be attributed to the low momentum region downstream of the turbine, due to possible flow separation and strong secondary flows, which are redistributed through the stator resulting in the higher axial velocities near $88 \%$ span. The distribution of mass averaged tangential velocity (Fig. 16) upstream of the stator shows uniform distribution from shell to $39 \%$ span, with an increase in $\overline{V_{\theta}}$, from this location to the core. Considerable redistribution takes place inside the stator due to differential blade turning, with higher change occurring near the shell and lower change near the core region. The $\overline{V_{\theta}}$

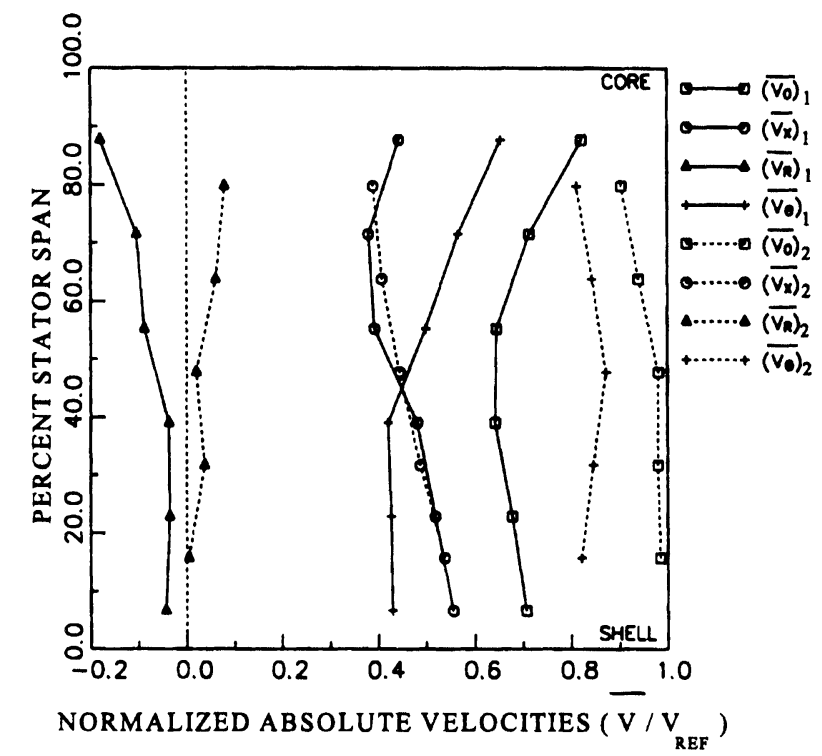

FIGURE 16 Radial Distribution of the Mass Averaged Absolute Normalized Velocity Components $\left(\bar{V} / V_{R E F}\right)$ at the Stator Inlet and Exit. 
distribution is more uniform downstream of the stator. The maximum change occurs near the mid-span region.

The radial distribution of the mass averaged yaw angles (Fig. 17) show very small flow turning near the core region and higher flow turning near the shell. The distribution of yaw angles downstream of stator shows small variation from the shell to the core. However, yaw angle distribution upstream of the stator shows large non-uniformity in the radial direction. The maximum variation from the shell to the core is approximately 18 degrees. As a result the flow field inside the stator blade varies substantially from the shell to the core. The radial distribution of pitch angles show radial inward flow at the inlet and radial outward flow at the exit of the stator. The magnitudes of the pitch angles are smaller near the shell and higher near the core. The higher pitch angles near the core downstream of the stator could be attributed to the differential bending of the streamlines as explained by Marathe, Lakshminarayana and Dong (1994).

\section{RADIAL DISTRIBUTION OF UNSTEADY FLOW PROPERTIES UPSTREAM OF THE STATOR}

Radial distribution of the rms values of each of the unsteady components, (periodic, aperiodic and unresolved) downstream of the turbine, (upstream of the stator) are presented in this section. The rms values are calculated by using the equations:

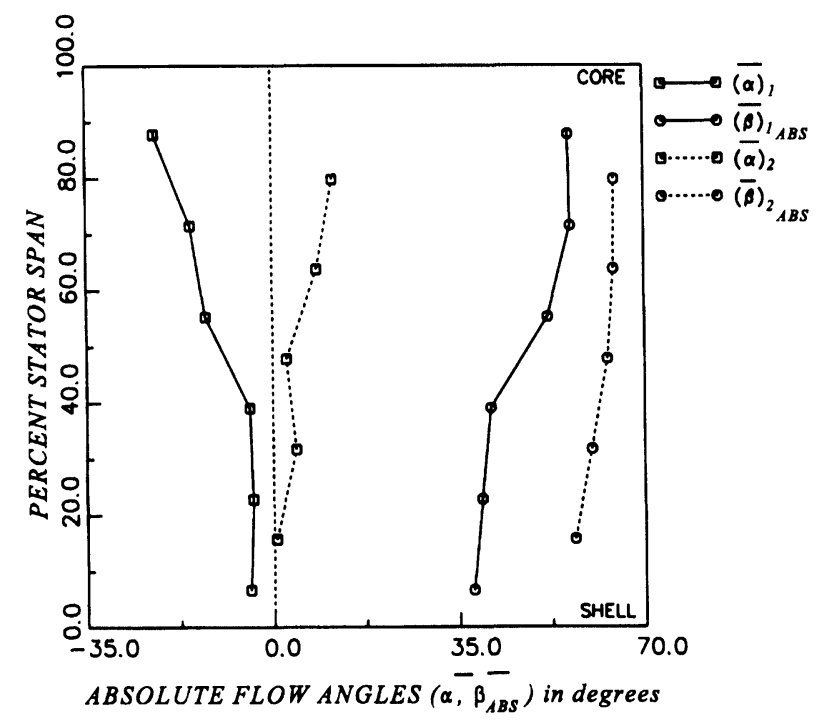

FIGURE 17 Radial Distribution of the Mass Averaged Absolute Flow Angles $\bar{\alpha}, \bar{\beta}_{A B S}$ ) at the Stator Inlet and Exit.

$$
\begin{aligned}
& \overline{\overline{\left(\hat{P}_{I}\right)_{R M S}}}=\sqrt{\frac{1}{N_{p p b}} \sum_{I=1}^{N_{p p b}}\left(\hat{P}_{I}\right)^{2}} \\
& \overline{\overline{\left(\tilde{P}_{I J}\right)_{R M S}}}=\sqrt{\frac{1}{N_{b p c}} \frac{1}{N_{p p b}} \sum_{J=1}^{N_{b p c}} \sum_{I=1}^{N_{p p b}}\left(\tilde{P}_{I J}\right)^{2} \text { and }} \\
& \overline{\overline{\left(P_{I J K}^{\prime}\right)_{R M S}}}=\sqrt{\frac{1}{N_{c y c}} \frac{1}{N_{b p c}} \frac{1}{N_{p p b}} \sum_{K=1}^{N_{c y c}} \sum_{J=1}^{N_{b p c}} \sum_{I=1}^{N_{p p b}}\left(P_{I J K}^{\prime}\right)^{2}}
\end{aligned}
$$

Where, $\mathrm{P}$ is a derived flow property $\left(\mathrm{V}, \alpha, \beta, \mathrm{P}_{0}, \mathrm{P}_{\mathrm{s}}\right.$ etc. $)$ and $\mathrm{N}$ is total number of points processed in a data set (typically 261000 points)

All the quantities presented here are in the absolute frame of reference. These quantities represent the unsteady flow properties perceived by the stator.

\section{Radial Distribution of rms of Unsteadiness in Absolute Stagnation Pressure}

The radial distribution of various components (periodic, aperiodic, unresolved) of absolute unsteady stagnation pressure coefficients are shown in Fig. 18. The total unsteadiness in stagnation pressure is approximately $23 \%$ of the reference dynamic head near the core and approximately $13 \%$ near the shell. However, the reference dynamic head $\left(Q_{\mathrm{REF}}=\rho \mathrm{V}_{\mathrm{REF}}^{2} / 2\right)$ is approximately $43 \%$ of the stagnation pressure drop in the turbine blade (calculated from one dimensional analysis). Hence, the rms value of total unsteadiness in the absolute stagnation

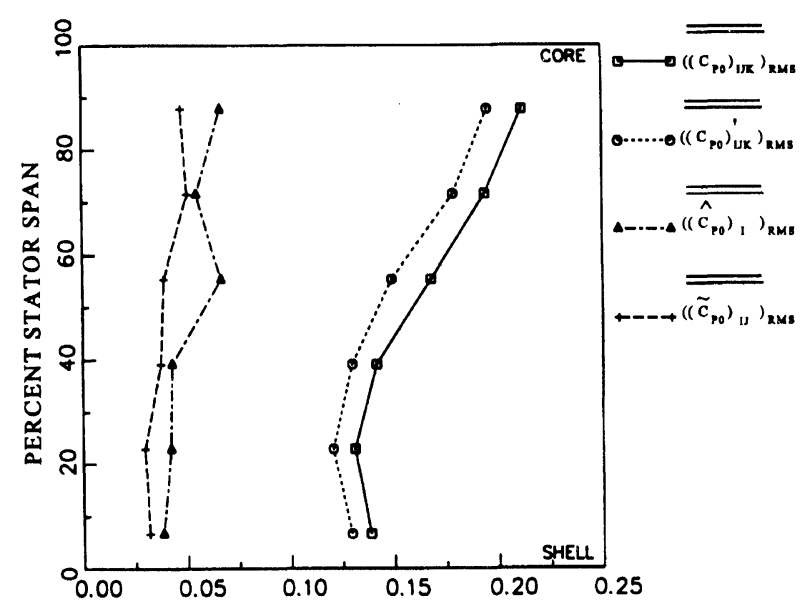
UNSTEADY ABSOLUTE STAGNATION PRESSURE COEFFICIENT FIGURE 18 Radial Distribution of rms of the Unsteady Components of Absolute Stagnation Pressure Coefficient $\overline{\overline{\left(\left(C_{P O}\right)_{A B S}\right)_{R M S}}}$ at the Stator Inlet. 
pressure coefficient downstream of the turbine is approximately $9.9 \%$ of the stagnation pressure drop in the turbine blade passage near the core and approximately $5.6 \%$ of the turbine stagnation pressure drop near the shell. The total unsteadiness is highest near the core. It decreases smoothly to $40 \%$ stator span and remains uniform from $40 \%$ stator span to shell. The higher magnitude of unsteadiness near the core is caused by flow separation and reattachment, within the turbine passage, which generates the low momentum region. The secondary flow will also contribute to total unsteadiness. This increases turbulence and hence the unresolved unsteadiness. The total unsteadiness includes the periodic fluctuations. However, the magnitude of periodic fluctuation is directly related to the tangential variation of the flow parameters inside the blade passage. Hence, nearly uniform distribution of the flow parameters across the passage from shell to mid-span (as discussed earlier in the paper) generates lower magnitudes of unsteadiness from the shell to the mid-span, whereas low momentum region and secondary flow regions observed from the mid-span to the core cause higher values of total unsteadiness. The unsteady flow field is dominated by unresolved unsteadiness (which includes turbulence) which is nearly $80 \%$ of total unsteadiness near the core. The periodic and aperiodic components contribute approximately $10 \%$ each to the total unsteadiness. It should be noted that the aperiodic component is generated by several sources periodic over one cycle but not at the blade passing frequency. As a result, the contribution of the aperiodic signal is significant. However, detailed study of aperiodic signals is beyond the scope of this paper. Therefore, only the radial distribution of the rms values will be presented.

The radial distribution of the periodic component of the unsteady stagnation pressure shows a rather smooth distribution except at $55 \%$ stator span, where a sudden change in the magnitude of the blade periodic component occurs. It should be noted that this change in magnitude coincides with the jump in the relative stagnation pressure and the axial velocity as discussed earlier in the paper. This suggests that the periodic component of the stagnation pressure increases in the region of flow separation and secondary flows as these regions induce high non-uniformity in tangential direction. This jump can also be attributed to the jump in the periodic fluctuations in the axial velocity at the same location. The distribution of rms value of the periodic component of the axial velocity is discussed later in the paper. The rms value of the aperiodic component shows nearly uniform distribution indicating that the radial variation of mean stagnation pressure does not influence aperiodic component.

\section{Radial Distribution of rms of Unsteadiness in Static Pressure}

The radial distribution of rms values of unsteady static pressure coefficient (Fig. 19) is significantly different from the radial distribution of the stagnation pressure coefficient. A sudden drop in the unresolved unsteadiness is observed at $55 \%$ stator span. This drop is attributed to the drop in the unresolved unsteadiness in total velocity as discussed later in the paper. The rms values of periodic component show non-uniformity from shell to core. A jump in the rms value is observed near the core. This jump can be attributed to high variation of static pressure in the tangential direction with higher static pressure at mid-passage and lower static pressures near the wakes. The distribution of $\mathrm{rms}$ of aperiodic unsteadiness is uniform from the core to the shell. This shows that the aperiodic components of the pressures are not affected by the non-uniform radial distribution of the flow parameters. This may be caused by the non-uniform passages of the pump and non-uniform flow at the inlet of the pump.

\section{Radial Distribution of the rms of the Velocity Components and the Yaw Angle}

The radial distribution of the rms value of the total unsteadiness in the total velocity (Fig. 20) is very similar to that of $\left(\mathrm{C}_{\mathrm{PS}}\right)_{\mathrm{RMS}}$. The values of unresolved unsteadi-

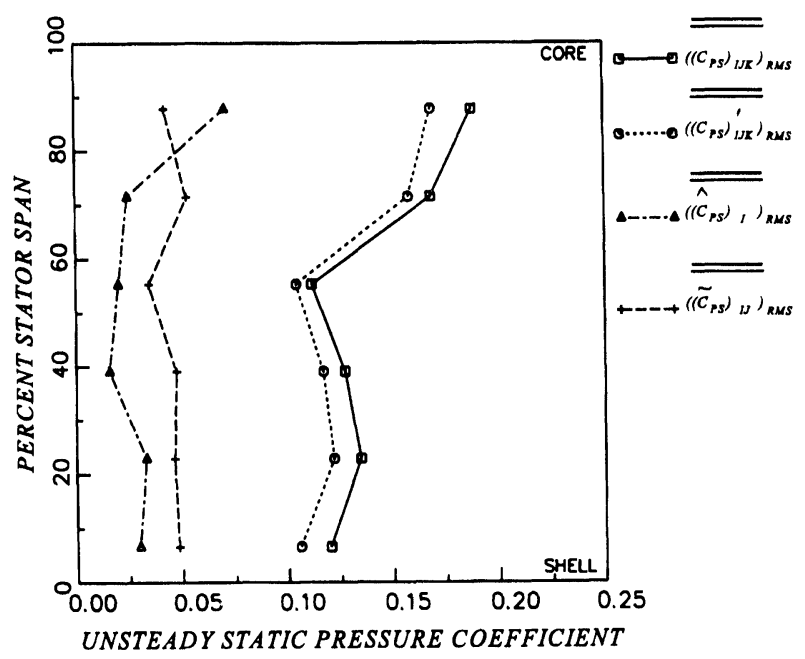

FIGURE 19 Radial Distribution of rms of the Unsteady Components of Static Pressure Coefficient $\overline{\overline{\left(C_{P S}\right)_{R M S}}}$ at the Stator Inlet. 


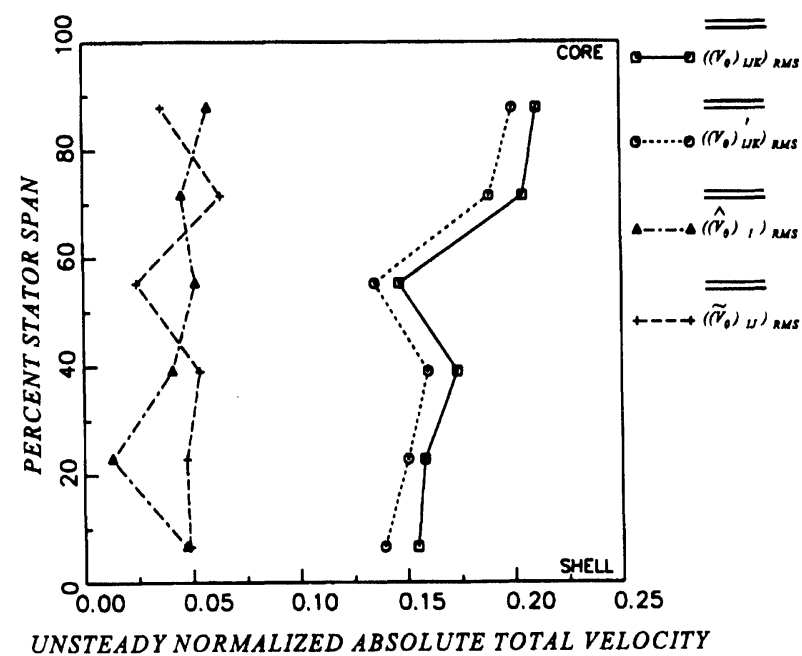

FIGURE 20 Radial Distribution of rms of the Unsteady Components of Absolute Normalized Total Velocity $\overline{\overline{\left(V_{O}\right)_{R M S}}} / V_{R E F}$, at the Stator Inlet.

ness are high (approx. 22\% of the mean total velocity, $\mathrm{V}_{01}$ ) near the core due to the reasons explained earlier. A sudden drop in the unresolved unsteady total velocity is observed at $55 \%$ stator span from the hub. This drop is attributed to the drop in the unresolved unsteadiness in the tangential velocity (not shown) at the same radial position. Both relative and absolute tangential velocity distribution (not shown) indicate a nearly uniform distribution from suction surface to pressure surface at $55 \%$ stator span. The maximum variation is less than $10 \%$ of the mean value. This causes low values of unsteadiness in periodic and unresolved unsteady components of tangential velocity. This significantly reduces the unresolved unsteadiness in total velocity at $55 \%$ stator span. The other types of mixed flow turbomachinery also exhibit low velocities near the convex shroud endwall due to the separation. The flow field near the convex side separates due to curvature and the adverse pressure gradient. This results in the higher losses and lower velocities near the convex endwall as observed by Khalil et al. (1976) and Johnson and Moore (1983). The influence of the endwall boundary layers on the flow field is more pronounced in the mixed flow turbomachinery than in the axial flow turbomachinery. As a result, the flow separation and reattachment of the flow near the core could result in high turbulence intensity and hence, high values of unresolved component. The contribution of the periodic and the aperiodic component of unsteadiness in total velocity is small (approx. 4-6\% of $\mathrm{V}_{01}$ each). The periodic component shows nearly uniform distribution from the core to the shell except at $22 \%$ of the stator span where the periodic component is very small. This non-uniformity can be attributed to the smaller rms value of the periodic component of the tangential velocity. The distribution of aperiodic component is non-uniform near the core and nearly uniform near the shell region. This type of distribution is also observed in aperiodic components of axial and tangential velocities. The radial distribution of unsteadiness in the tangential velocities were found similar to those of the total velocity.

The radial distribution of passage averaged unsteady components of axial velocity (Fig. 21) is significantly different to that of total velocity. All the unsteady components (periodic, aperiodic, unresolved and total) show nearly uniform distribution from $7 \%$ stator span to $39 \%$ stator span. The magnitude of unresolved, aperiodic and total unsteadiness increases smoothly from 39\% stator span to $71 \%$ stator span and decreases slightly from $71 \%$ stator span to $88 \%$ stator span. However, a sudden jump in the periodic unsteadiness is observed from $39 \%$ stator span to $55 \%$ stator span. This jump is caused by the presence of the low velocity zone near the core as discussed earlier in the paper.

The distribution of the rms values of yaw angles (Fig. 22) show small variations in the rms values from core to shell. The unresolved unsteadiness dominates the total unsteadiness. The rms of periodic unsteadiness is small ( $3^{\circ}$ near the shell and $7^{\circ}$ near the core). However, this unsteadiness would change the incidence angle by $\pm 5^{\circ}$ near the shell and $\pm 10^{\circ}$ near the core. This would affect

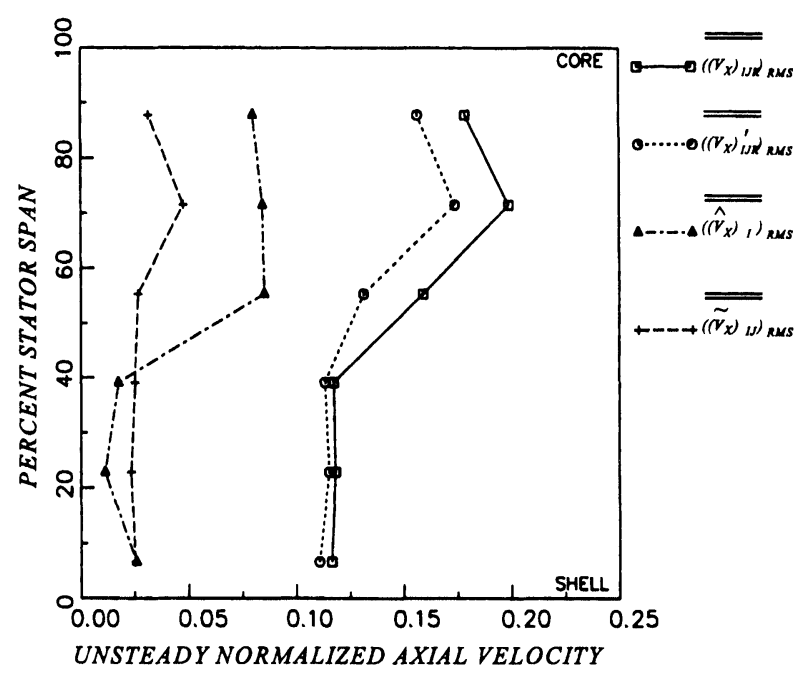

FIGURE 21 Radial Distribution of rms of the Unsteady Components of Normalized Axial Velocity $\overline{\overline{\left(V_{X}\right)_{R M S}}} / V_{R E F}$ at the Stator Inlet. 
the stator flow field significantly. The maximum tangential variation in the yaw angle is found to be $\pm 14^{\circ}$ near the core, $\pm 15^{\circ}$ near the mid-span and $\pm 10^{\circ}$ near the shell. It should be noted that the radial distribution of periodic unsteadiness in the yaw angle is similar to the radial distribution of the periodic unsteadiness of the axial velocity. Since, the variation of tangential velocity from pressure surface to the suction surface (not shown) is significant only at $7 \%, 39 \%$ and $88 \%$ of the stator span, the variations of the yaw angle at other radii are caused by the axial velocity variation at those radii.

Large periodic fluctuations in the radial velocity (Fig. 23) generate large fluctuations in the pitch angle (not shown). The rms values of the overall unsteady pitch angle are as high as 35 degrees near the core and 10 degrees near the shell. It should be noted that the pitch angles are calculated with reference to the axial direction. The distribution shows that the contribution of the periodic component to total unsteadiness is high (approx. $25 \%$ near shell and approx. 60-75\% of near core). This is an important observation since the overall unsteadiness is dominated by unresolved unsteadiness in all other flow parameters. In the region of strong secondary flow ( $71 \%$ span) the periodic component is higher than the unresolved component. This could be attributed to the high tangential gradient in the radial velocity in the secondary flow region. The aperiodic component is relative small ( approx. $8-10 \%$ of $\mathrm{rms}$ value of total unsteadiness) and distribution of aperiodic component is nearly uniform from core to shell except in the region of secondary flow where aperiodic component is higher.j

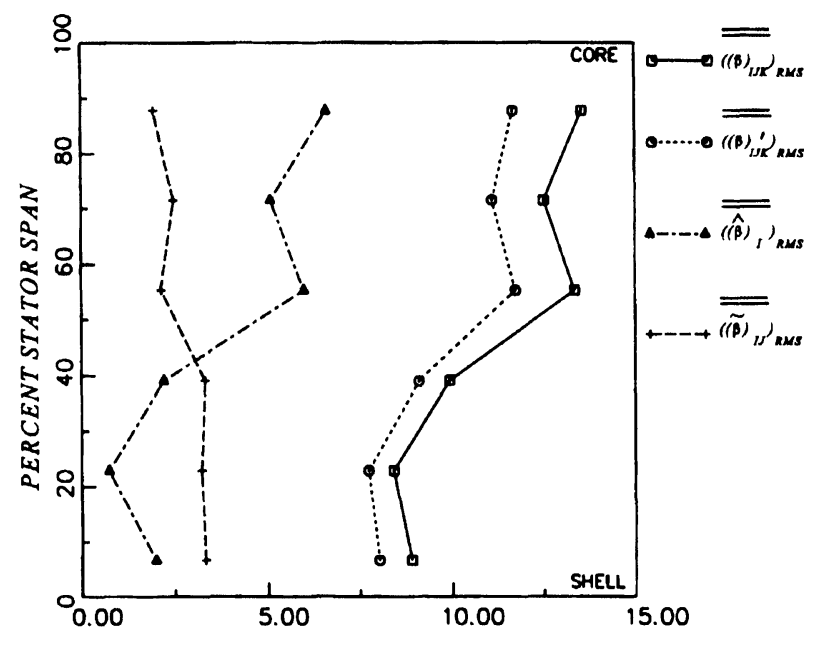

UNSTEADY ABSOLUTE YAW ANGLE (in degrees)

FIGURE 22 Radial Distribution of rms of the Unsteady Components of Absolute Yaw Angles $\overline{\overline{\left((\beta)_{A B S}\right)_{R M S}}}$ at the Stator Inlet.

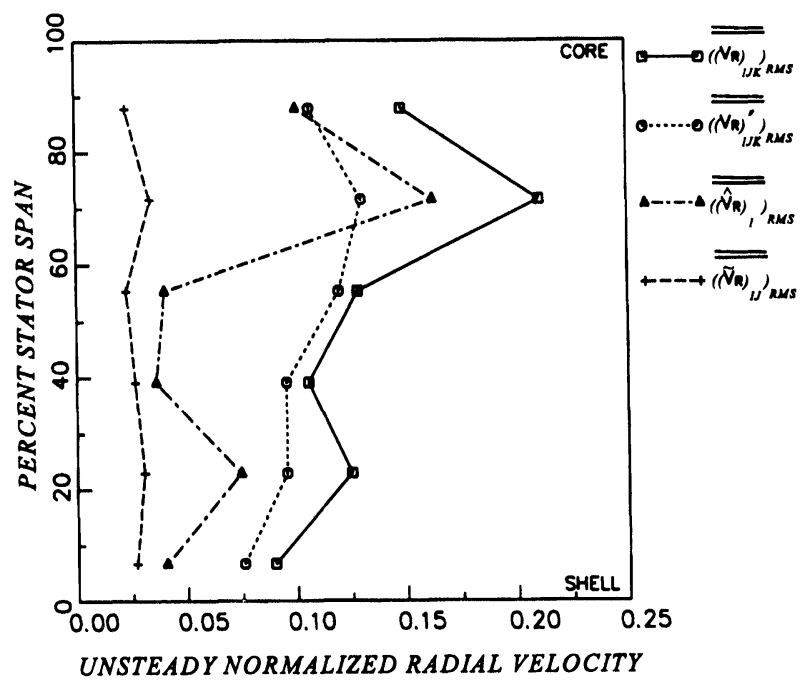

FIGURE 23 Radial Distribution of rms of the Unsteady Components of Normalized Radial Velocity $\overline{\overline{\left(V_{R}\right)_{R M S}}} / V_{R E F}$ at the Stator Inlet.

The radial distribution of the unresolved component of the unsteady radial velocity is different from those observed for other components (Fig. 21). The unresolved component is nearly-uniform from core to the mid-span and reduces gradually from mid-span to shell. Also the magnitudes of the unresolved unsteadiness are smaller than those observed for other unsteady velocity components. However, it should be noted that the magnitude of the total unsteadiness in the radial velocity is similar to that observed for other components of velocity, and pressure.

\section{CONCLUSIONS}

The distribution of flow properties at the exit of the turbine of an automotive torque converter, in the relative reference frame, shows a very non-uniform flow field near the core and nearly uniform flow field near the shell. The blade wakes are thin and the wake decay is rapid near the shell. A velocity profile similar to a fullydeveloped flow profile (in blade-to-blade direction) is observed at all radii, which is more pronounced near the core. A small zone of low momentum fluid is observed near the core suction surface due to possible flow separation and reattachment of the flow inside the turbine passage. The static pressure varies significantly near the core, with lower static pressures inside the wake and higher static pressures near the mid-passage. However, the radial distribution of mass averaged static pressure is smooth from the shell to the core indicating that all the radial variations in the mass averaged relative and 
absolute stagnation pressures are caused only by the radial variations of the mass averaged velocities.

A secondary vortex, observed near the core, results in lower stagnation pressure, lower static pressure and lower relative velocities in this region. The yaw and the pitch angles near the core are strongly influenced by the vortex. The secondary flow is weak near the shell and is swept away by the underturning flow. The influence of secondary flow near the shell is negligible. However, it influences the periodic components of unsteady velocities.

Each instantaneous unsteady flow property was divided into four components; time mean, periodic, aperiodic and unresolved. The unresolved unsteadiness of all properties dominates the unsteady flow field, which is consistent with the data reported by Brun (1993). The unresolved unsteadiness in the velocity at the stator inlet is high (approx. 20\% of the mean value) near the core and moderate near the shell (approx. 14\% of the mean value). These magnitudes indicate very high levels of turbulence at both the core and the shell regions. The periodic component generated due to the blade-to-blade distribution of the steady state flow properties in the turbine reference frame is significant. Due to closed loop circuit path and close spacing of the blade row, the pump flow field influences the unsteady component at the inlet to the turbine generating unsteadiness at frequencies which are combinations of the blade passing frequencies. As a result, the aperiodic component is also significant. Although, the fluctuations of radial component of the velocity are highest among the three components, the unsteadiness in the tangential velocity and static pressure show maximum influence on the unsteady total velocity and stagnation pressure.

The radial distribution of flow properties upstream and downstream of the stator indicates higher losses near the core and the shell and slight increase in the stagnation pressure near the mid-span. High losses are caused by the thick boundary layer near the shell suction surface and due to the low momentum region near the core whereas the slight rise in the stagnation pressure is due to the redistribution of the stagnation pressure inside the stator. The radial distribution of the static pressure is influenced not only by the swirl velocity but also by the meridional streamline curvature. The distribution of the axial velocity at the inlet and the exit is identical except in the region of low momentum fluid where a redistribution of axial velocity is observed. The distribution of radial velocity at the inlet of the stator is also similar to that at the exit. However, the radial distribution of the tangential velocity changes significantly, from inlet to the exit of the stator, due to higher flow turning (approx. $20^{\circ}$ ) near the shell and lower flow turning (approx. $3^{\circ}$ ) near the core, inside the stator passage.

It can be concluded that the flow field inside a torque converter is highly three dimensional, viscous and unsteady. Hence, the effects of three dimensionality and unsteady effects should be incorporated in the design of torque converter elements to achieve improved performance.

\section{Acknowledgements}

This work was sponsored by Powertrain Division of General Motors Corporation. The authors wish to express their gratitude to D. Maddock and R. By for their assistance, advice and comments on the experimental program. Assistance by $\mathrm{Y}$. Dong in the data acquisition is also acknowledged.

\section{Nomenclature}

$C$

$\left(C_{P O}\right)_{R E L}$

$\left(C_{P O}\right)_{A B S}$

$C_{P S}$

$H$

$N_{C Y C}$

$N_{B P C}$

$N_{P P B}$

$P$

$P_{H U B}$

$P S, S S$

$Q_{R E F}$

$R$

$S$

$S R$

$T R$

$U$

V

$V_{R E F}$

$\overline{V_{x}}$

W

$\alpha$

$\beta$

$\rho$

()$_{R M S}$

\section{Subscripts}

1,2

$0, S$

$A B S$

$R E L$

$X, R, \theta$

hub, tip

Max
Chord length

$2\left(\left(\mathrm{P}_{0}\right)_{\mathrm{REL}}-\mathrm{P}_{\mathrm{HUB}}\right) / \rho \mathrm{V}_{\mathrm{REF}}^{2}$

$2\left(\left(\mathrm{P}_{0}\right)_{\mathrm{ABS}}-\mathrm{P}_{\mathrm{HUB}}\right) / \rho \mathrm{V}^{2}{ }_{\mathrm{REF}}$

$2\left(\mathrm{P}_{\mathrm{S}}-\mathrm{P}_{\mathrm{HUB}}\right) / \rho \mathrm{V}^{2}{ }_{\text {REF }}$

Spanwise distance from turbine shell (hub) normalized by the turbine blade span $(\mathrm{H}=0$ at the shell, 1 at the core)

Number of cycles in a data set

Number of turbine passages in a cycle

Number of data points per turbine passage

Pressure normalized by $\rho \mathrm{V}^{2}{ }_{\mathrm{REF}} / 2$

Reference pressure on the hub

Pressure and suction side

Reference dynamic head $\rho \mathrm{V}_{\text {REF }}^{2} / 2$

Radius

Blade spacing

Speed ratio (turbine rpm/pump rpm)

Torque ratio (turbine torque/pump torque)

Blade speed

Absolute Velocity normalized by $\mathrm{V}_{\mathrm{REF}}$

Normalizing Velocity

$\left(U_{\text {TIP PUMP }} \sqrt{1-\mathrm{SR})}\right)$

Mass averaged axial velocity downstream of the turbine

Relative Velocity normalized by $\mathrm{V}_{\mathrm{REF}}$

Pitch angle, $\tan ^{-1}\left(\mathrm{~V}_{\mathrm{R}} / \mathrm{V}_{\mathrm{X}}\right)$

Yaw angle (measured from the axial direction) $\tan ^{-1}\left(\mathrm{~V}_{\theta} / \mathrm{V}_{\mathrm{X}}\right)$

Density of the fluid

The root mean square values of fluctuating quantities $\left(\mathrm{V}, \mathrm{P}_{0}, \mathrm{P}_{\mathrm{S}}, \alpha, \beta\right)$

Inlet and exit of the stator

Total/Stagnation, static

Absolute (stator) reference frame

Relative (turbine) reference frame

Axial, radial and tangential components

Property at the hub and tip, respectively

Maximum value 
$R M S$

$I, J, K$

Root mean square value

Subscripts defining data point number inside a blade, blade number in a cycle and cycle number respectively. (Typically $\mathrm{I}=1-50, \mathrm{~J}=1$ $29, \mathrm{~K}=1-50$ )

\section{Superscripts}

Passage (mass) averaged value (Equation 2) Passage-to-passage periodic component (Equation 3)

Passage-to-passage aperiodic component (Equation 4)

Unresolved Component (Equation 6)

Passage Averaged Value

\section{References}

Bahr, H. M. et al., 1990, "Laser Velocimeter Measurements in the Stator of a Torque Converter," SAE Paper 901769.

Browarzik V. and Grahl K. G., 1992, "Non-Steady Flow Measurements Inside a Hydrodynamic Torque Converter by Hot-film Anemometry," ASME Paper 92-GT-161

Brun, K., 1993, "Laser Velocimetry Measurements in a Turbine of an Automotive Torque Converter," Master's Thesis, University of Virginia.

Brun, K., Flack, R. D., and Gruver, J. K., 1994, "Laser Velocimeter Measurements in the Pump of a Torque Converter Part II-Unsteady Measurements," ASME Paper 94-GT-48.

By, R. R. and Lakshminarayana, B., 1991, "Static Pressure Measurements in a Torque Converter," SAE Paper 911934.

By, R. R. and Lakshminarayana, B., 1995, "Measurement and Analysis of Static Pressure Field in a Torque Converter Pump," 2nd ASME Pumping Machinery Symposium, FED-Vol. 154, pp 253-264, Journal of Fluids Engineering, Transactions of ASME, Vol 117 pp. 109-115.

By, R. R., Kunz, R. F., Lakshminarayana, B., 1995, "Navier-Stokes Analysis of the Pump Flow Field of an Automotive Torque Converter," Second ASME Pumping Machinery. Symposium, FED-Vol. 154, pp. 265-274, Journal of Fluids Engineering, Transactions of ASME, Vol 117, pp. 116-122.

Gruver, J. K., Flack, R. D., and Brun, K., 1994, "Laser Velocimeter Measurements in the Pump of a Torque Converter Part I-Average Measurements," ASME Paper 94-GT-47.

Johnson, M. W. and Moore, J., 1983, "Secondary Flow Mixing Losses in a Centrifugal Impeller," Journal of Engineering for Power, Transactions of ASME, Vol. 105, pp. 24-32.

Khalil, I. M. et. al., 1976, "Losses in Radial Inflow Turbines," Journal of Fluids Engineering, Transactions of ASME, September, pp. 364-373.

Lakshminarayana, B. and Horlock, J. H., 1973, "Generalized Expressions for Secondary Vorticity Using Intrinsic Coordinates," Journal of Fluid Mechanics, Vol. 59, pp. 97-115.

Marathe. B. V., Lakshminarayana, B., Dong, Y., 1994, "Experimental and Numerical Investigation of Stator Exit Flow Field of an Automotive Torque Converter," ASME Paper 94-GT-32.

Suder K. L. et. al., 1987, "Measurements of the Unsteady Flow Field Within the Stator Row of a Transonic Axial Flow Fan I-Measurement and Analysis Technique.," ASME Paper 87-GT-226.

Treaster, A. L. And Yocum, A. M., 1979, "The Calibration and Application of Five-Hole Probes," ISA Transactions, Vol. 18, No. 3, pp. 23-34.

Zaccaria, M. and Lakshminarayana, B., 1995, "Investigation of ThreeDimensional Flow Field at the Exit of a turbine Nozzle," Journal of Propulsion and Power, vol. 11, p. 55. 

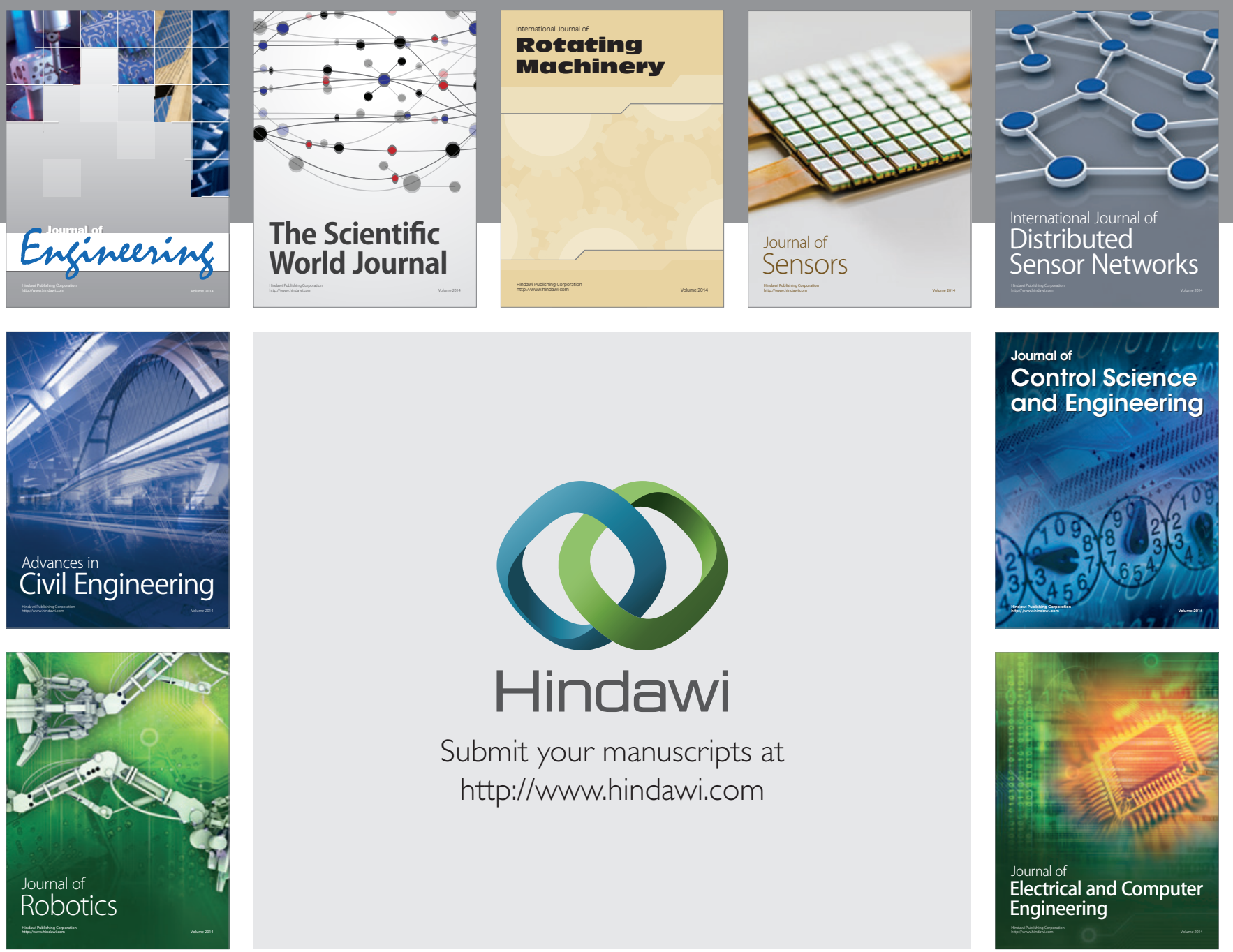

Submit your manuscripts at

http://www.hindawi.com
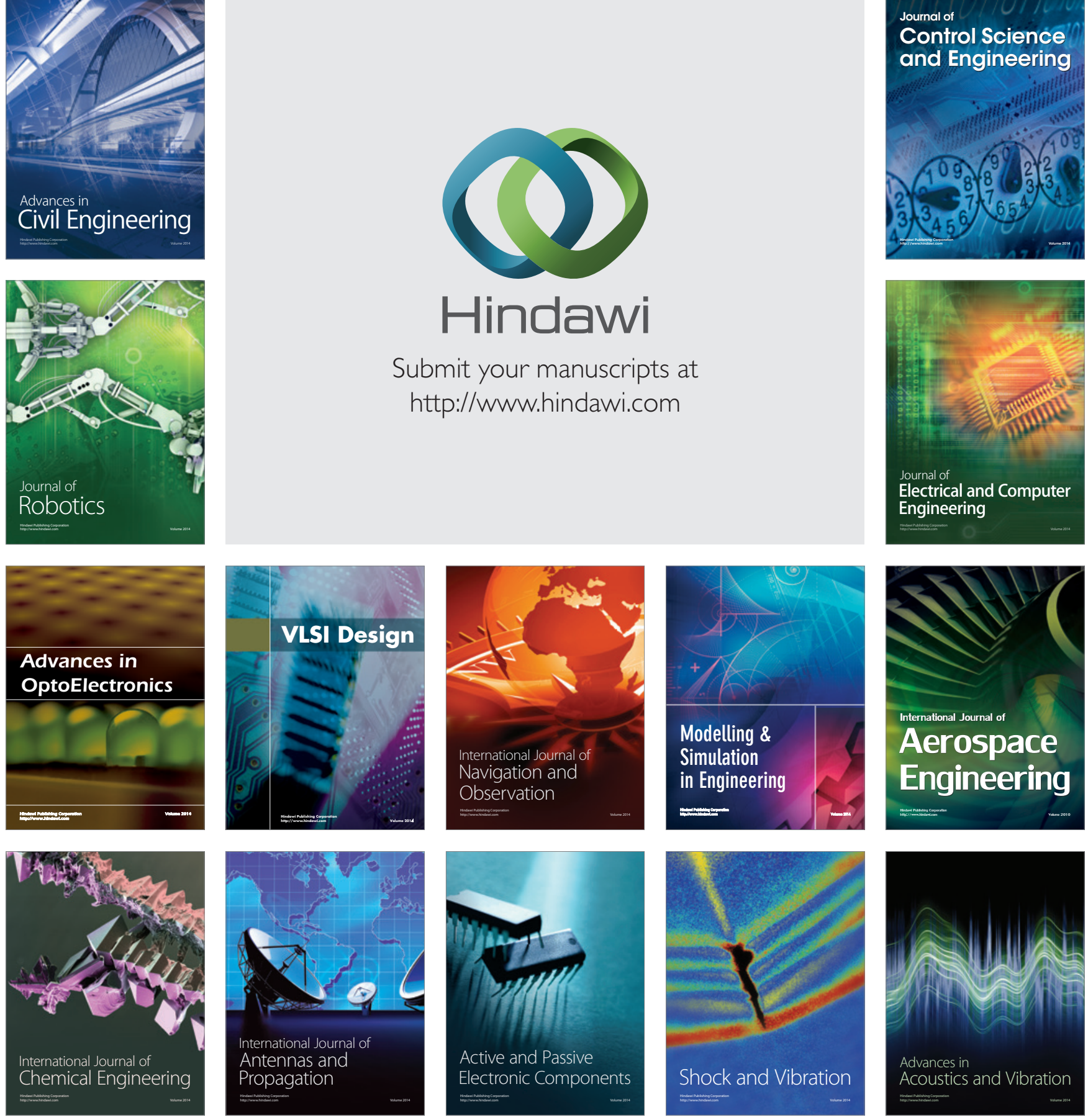